Research Article Special Issue: Combinatorial Matrices

Open Access

\author{
A. Carmona*, A.M. Encinas, and M. Mitjana
}

\title{
A combinatorial expression for the group inverse of symmetric $M$-matrices
}

https://doi.org/10.1515/spma-2020-0137

Received October 30, 2020; accepted April 15, 2021

\begin{abstract}
By using combinatorial techniques, we obtain an extension of the matrix-tree theorem for general symmetric $M$-matrices with no restrictions, this means that we do not have to assume the diagonally dominance hypothesis. We express the group inverse of a symmetric $M$-matrix in terms of the weight of spanning rooted forests. In fact, we give a combinatorial expression for both the determinant of the considered matrix and the determinant of any submatrix obtained by deleting a row and a column. Moreover, the singular case is obtained as a limit case when certain parameter goes to zero. In particular, we recover some known results regarding trees. As examples that illustrate our results we give the expressions for the Group inverse of any symmetric $M$-matrix of order two and three. We also consider the case of the cycle $C_{4}$ an example of a non-contractible situation topologically different from a tree. Finally, we obtain some relations between combinatorial numbers, such as Horadam, Fibonacci or Pell numbers and the number of spanning rooted trees on a path.
\end{abstract}

Keywords: matrix-tree theorem, spanning tree, group inverse, effective resistances, Schrödinger operator, symmetric $M$-matrices

MSC: 05C05, 15A09, 15A10

\section{Introduction}

The classical matrix-tree theorem, whose first version was proved by G. Kirchhoff in 1847, relates the principal minors of the Laplacian matrix of a network $\Gamma$, with the total weight of spanning trees of $\Gamma$. Therefore, it refers to singular, symmetric and diagonally dominant $M$-matrices. Since then, many generalizations and different proofs have been considered (see for instance [1] and the references therein). In particular, the matrix-tree theorem has been extended to the case of non-singular, symmetric, $\alpha$-diagonally dominant $M$-matrices, see $[12,16,26,27]$. By $\alpha$-diagonally dominant $M$-matrix, we understand those diagonally dominant $M$-matrices that have equal excess, $\alpha$, in the diagonal. Hence, the value of the determinant is expressed as a polynomial in $\alpha$ whose coefficients are given by the weight of spanning rooted forest. In [21], the case of non-symmetric and (non-singular) Laplacian matrices is consider for the case of complex weights. In general, it is not an easy task to compute the number of spanning trees together with their weights in the weighted case, see [25] for some explicit expression in the case that the conductances are tensorial product and the underlying graph is structured. In [28] we found expressions for enumerating spanning trees in bipartite graphs, line graphs, generalized line graphs, middle graphs, total graphs, generalized join graphs and vertex-weighted graphs.

\footnotetext{
^Corresponding Author: A. Carmona: Departament de Matemàtiques, Universitat Politècnica de Catalunya, 08039 Barcelona, Spain, E-mail: angeles.carmona@upc.edu

A.M. Encinas: Departament de Matemàtiques, Universitat Politècnica de Catalunya, 08039 Barcelona, Spain, E-mail: andres.marcos.encinas@upc.edu

M. Mitjana: Departament de Matemàtiques, Universitat Politècnica de Catalunya, 08039 Barcelona, Spain, E-mail: margarida.mitjana@upc.edu
} 
In this work we obtain an extension of the matrix-tree theorem for general symmetric $M$-matrices; which means that we remove the diagonally dominance hypothesis. The techniques we use are of combinatorial nature. Specifically, to prove our results we use an extension of the undirected version of the all-minors theorem by S. Chaiken [11], see also [8] for a beautiful application. We give a combinatorial expression for both the determinant of the considered matrix and the determinant of any submatrix obtained by deleting a row and a column. Thus, we express the group inverse of a symmetric $M$-matrix in terms of the weight of spanning rooted forests. Moreover, the singular case is obtained as a limit case when the parameter goes to zero and we recover some known result regarding trees.

Given a finite set $V$, the set of real valued functions on $V$ is denoted by $\mathcal{C}(V)$. The standard inner product on $\mathcal{C}(V)$ is denoted by $\langle\cdot, \cdot\rangle$ and hence, if $u, v \in \mathcal{C}(V)$, then $\langle u, v\rangle=\sum_{x \in V} u(x) v(x)$. For any $x \in V, \varepsilon_{x} \in \mathcal{C}(V)$ stands for the Dirac function at $x$ and 1 is the function defined by $1(x)=1$, for any $x \in V$. On the other hand, $\omega \in \mathcal{C}(V)$ is called a weight if it satisfies that $\omega(x)>0$ for any $x \in V$ and moreover $\langle\omega, \omega\rangle=n=|V|$. The set of weights on $V$ is denoted by $\Omega(V)$.

The triple $\Gamma=(V, E, c)$ denotes a finite network; that is, a finite connected graph without loops nor multiple edges, with vertex set $V$, whose cardinality equals $n$, and edge set $E$, in which each edge $\{x, y\}$ has been assigned a conductance $c(x, y)>0$. So, the conductance can be considered as a symmetric function $c: V \times V \longrightarrow[0,+\infty)$ such that $c(x, x)=0$ for any $x \in V$ and moreover, vertex $x$ is adjacent to vertex $y$ iff $c(x, y)>0$. If we label the set of vertices, then functions can be identified with vectors in $\mathbb{R}^{n}$ and endomorphisms of $\mathcal{C}(V)$ with matrices of order $n$.

The combinatorial Laplacian or simply the Laplacian of the network $\Gamma$ is the endomorphism of $\mathcal{C}(V)$ that assigns to each $u \in \mathcal{C}(V)$ the function

$$
\mathcal{L}(u)(x)=\sum_{y \in V} c(x, y)(u(x)-u(y)), \quad x \in V .
$$

The matrix associated with $\mathcal{L}$ is always a singular symmetric $M$-matrix. Recall that a symmetric matrix $M$ is called a $M$-matrix if its off diagonal entries are non-positive, and its eigenvalues are non-negative. Moreover, a matrix $M$ is called irreducible if it is not similar via a permutation to a block upper triangular matrix. Therefore, a symmetric matrix is irreducible if and only if its associated graph is connected.

Given $q \in \mathcal{C}(V)$, the Schrödinger operator on $\Gamma$ with potential $q$ is the endomorphism of $\mathrm{e}(V)$ that assigns to each $u \in \mathcal{C}(V)$ the function $\mathcal{L}_{q}(u)=\mathcal{L}(u)+q u$, where $q u \in \mathcal{C}(V)$ is defined as $(q u)(x)=q(x) u(x)$; see for instance $[2,6]$.

Given a weight $\omega \in \Omega(V)$, we called Doob-potential determined by $\omega$ the function given by $q_{\omega}=$ $-\omega^{-1} \mathcal{L}(\omega)$. It is well-known that any Schrödinger operator is self-adjoint and moreover it is positive semidefinite iff there exist $\omega \in \Omega(V)$ and $\lambda \geq 0$ such that $q=q_{\omega}+\lambda$; see [2]. In addition, $\mathcal{L}_{q}$ is singular iff $\lambda=0$, in which case $\left\langle\mathcal{L}_{q_{\omega}}(v), v\right\rangle=0$ iff $v=a \omega, a \in \mathbb{R}$. In any case, $\lambda$ is the lowest eigenvalue of $\mathcal{L}_{q}$ and its associated eigenfunctions are multiple of $\omega$. In [4], some of the authors proved that any irreducible, symmetric $M$-matrix can be identified with a positive semi-definite Schrödinger operator on a network.

In this work we fix $\omega \in \Omega(V)$ and for each $\lambda \geq 0$, we consider the positive semi-definite Schrödinger operator $\mathcal{L}_{q}$, where $q=q_{\omega}+\lambda$. Then, $\mathcal{L}_{q}$ is an automorphism on $\omega^{\perp}$, whose inverse is called (orthogonal) Green operator and is denoted by $\mathcal{G}_{q}$. We can extend the Green operator to $\mathcal{C}(V)$ by assigning to any $f \in \mathcal{C}(V)$, the unique $u \in \mathcal{C}(V)$ such that $\mathcal{L}_{q}(u)=f-\frac{1}{n}\langle\omega, f\rangle \omega$ and $\langle u, \omega\rangle=0$. The Green operator is self-adjoint, singular and satisfies that $\mathcal{G}_{q}(\omega)=0$, see [4].

The function $G_{q}: V \times V \longrightarrow \mathbb{R}$, defined as $G_{q}(x, y)=\mathcal{G}_{q}\left(\varepsilon_{y}\right)(x)$, for any $x, y \in V$, is called Green function. Moreover, for $\lambda=0$, the matrix associated with $G_{q}$ is the Moore-Penrose inverse of $\mathcal{L}_{q}$ (that in this case coincides with the group inverse); whereas, for $\lambda>0$, we have that $\mathcal{L}_{q}^{-1}=\mathcal{G}_{q}+\frac{1}{n \lambda} \omega \otimes \omega$.

Through the manuscript, we use a common technique in the context of electrical networks and Markov chains, see [17, 18]. The authors considered this technique in [4] in order to generalize the Fiedler characterization of irreducible, symmetric and diagonally dominant $M$-matrices as resistive inverses, see [20], to all irreducible and symmetric $M$-matrices or equivalently, to all positive semi-definite Schrödinger operators. 


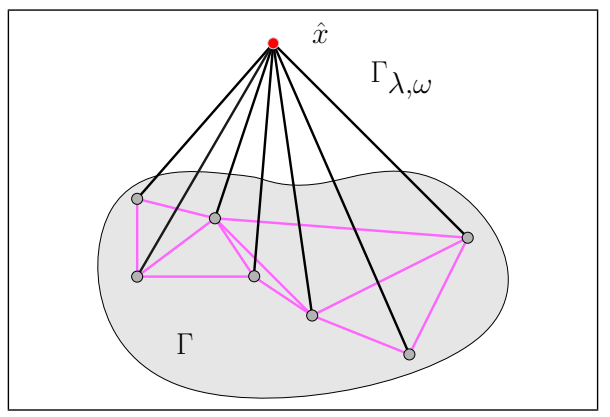

Figure 1: Host network.

Given a positive-definite Schrödinger operator on $\Gamma, \mathcal{L}_{q}$, the method consists in embedding the given network into a suitable host network. The new network is constructed by adding a new vertex, that represents a grounded vertex or an absorbing state in the context of Markov chains. The new vertex, $\hat{x}$, is joined with each vertex in the original network through a new edge whose conductance is the diagonal excess of the original $M$-matrix after the application of the Doob transform; see Figure 1.

Given $\lambda>0, \omega \in \Omega(V)$ and $\hat{x} \notin V$, we define the host network, $\Gamma_{\lambda, \omega}=\left(V \cup\{\hat{x}\}, c_{\lambda, \omega}\right)$ where $c_{\lambda, \omega}(x, y)=$ $c(x, y)$ when $x, y \in V$ and $c_{\lambda, \omega}(\hat{x}, x)=c_{\lambda, \omega}(x, \hat{x})=\lambda \omega(x)$ for any $x \in V$. We denote by $\mathcal{L}^{\lambda, \omega}$, its combinatorial Laplacian and by $\widehat{\omega} \in \Omega(V \cup\{\hat{x}\})$ the weight given by $\widehat{\omega}(x)=\omega(x)$ when $x \in V$ and $\widehat{\omega}(\hat{x})=1$.

The next result, whose proof can be found in [4, Proposition 2.8], establishes the relationship between the original Schrödinger operator $\mathcal{L}_{q}$ and a new semidefinite Schrödinger operator on $\Gamma_{\lambda, \omega}$.

Proposition 1.1. If $q=q_{\omega}+\lambda$ and we define $\hat{q}=-\frac{1}{\widehat{\omega}} \mathcal{L}^{\lambda, \omega}(\widehat{\omega})$, then $\hat{q}(\hat{x})=\lambda(n-\langle\omega, 1\rangle)$ and $\hat{q}=q-\lambda \omega$ on $V$. Moreover, for any $u \in \mathcal{C}(V \cup\{\hat{x}\})$ we get that $\mathcal{L}_{\hat{q}}^{\lambda, \omega}(u)(\hat{x})=\lambda\left(n u(\hat{x})-\left\langle\omega, u_{\left.\right|_{V}}\right\rangle\right)$ and

$$
\mathcal{L}_{\hat{q}}^{\lambda, \omega}(u)=\mathcal{L}_{q}\left(u_{\mid V}\right)-\lambda \omega u(\hat{x}) \text { on } V .
$$

\section{Matrix Tree Theorem for symmetric $M$-matrices}

In this section we aim to present the matrix tree-theorem for positive definite Schrödinger operators and to get the relation between the number of rooted forests and the Green function of the network. Moreover, taking limits when $\lambda$ goes to 0 we get the matrix tree-theorem for positive semi-definite Schrödinger operators.

If $\mathcal{T}$ is a network such that for each pair $x$ and $y$ of vertices, there is exactly one path joining $x$ and $y$, then $\mathcal{T}$ is called a weighted tree. Therefore, $\mathcal{T}$ is a weighted tree iff it has no cycles. Given $\Gamma$ a connected network, we say that $\mathcal{T}$ is a spanning tree of $\Gamma$ if both networks have the same set of vertices and each edge of $\mathcal{T}$ is also an edge of $\Gamma$ with the same weight. A forest is a non-necessarily connected network without cycles. A rooted forest is a forest with one vertex marked as a root in each connected component. Moreover a $k$-rooted forest is a forest with $k$ components and a vertex marked as a root in each connected component.

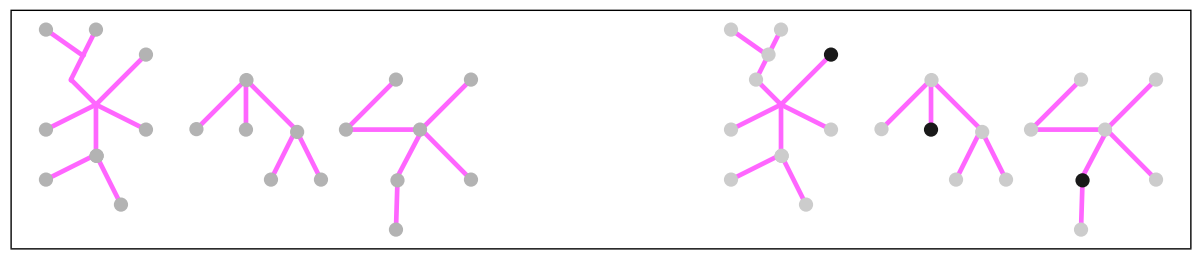

Figure 2: A forest (left) and a 3-rooted forest (right). 
The set of vertices and the set of edges of a forest $\mathcal{F}$, will be denoted by $V(\mathcal{F})$ and $E(\mathcal{F})$, respectively. The weight of a forest $\mathcal{F}$ is defined as the product of all weights of the edges of $\mathcal{F}$ taking into account the weights of the vertices given by $\omega$; i.e.,

$$
w(\mathcal{F})=\frac{\prod_{e \in E(\mathcal{F})} w(e)}{\prod_{x \in V(\mathcal{F})} \omega^{2}(x)},
$$

where $e=\{x, y\} \in E(\mathcal{F})$ and $w(e)=c(x, y) \omega(x) \omega(y)$ and we take the convention that the empty product equals 1.

We denote by $\mathcal{F}=\mathcal{F}(\Gamma)$ the set of rooted spanning forests of $\Gamma$ and $\mathcal{F}_{k}=\mathcal{F}_{k}(\Gamma)$ the set of $k$-rooted spanning forest of $\Gamma$. Moreover, $\mathcal{F}_{x}=\mathcal{F}_{x}(\Gamma)$ denotes the set of rooted spanning forests of $\Gamma$ such that $x$ is a root and $\mathcal{F}_{k, x}=\mathcal{F}_{k, x}(\Gamma)$ denotes the set of $k$-rooted spanning forests of $\Gamma$ such that $x$ is a root. Finally, we denote by $\mathcal{F}_{x, y}=\mathcal{F}_{x, y}(\Gamma)$ the set of rooted spanning forests of $\Gamma$ such that $x$ is a root and $y$ belongs to the same component as $x$ and $\mathcal{F}_{k, x, y}=\mathcal{F}_{k, x, y}(\Gamma)$ the set of $k$-rooted spanning forest of $\Gamma$ such that $x$ is a root and $y$ belongs to the same component as $x$. On the other hand, $\mathcal{F}_{k, x}^{\{y\}}$ denotes the set of rooted spanning forest of $\Gamma$ such that $x$ is a root and $y$ does not belong to the same component as $x$. If $\mathcal{F} \in \mathcal{F}_{k}$ we denote by $r(\mathcal{F})$ the set of its roots whereas if $\mathcal{F} \in \mathcal{F}_{k, x}$ we denote by $r^{*}(\mathcal{F})$ the set of roots different from $x$.

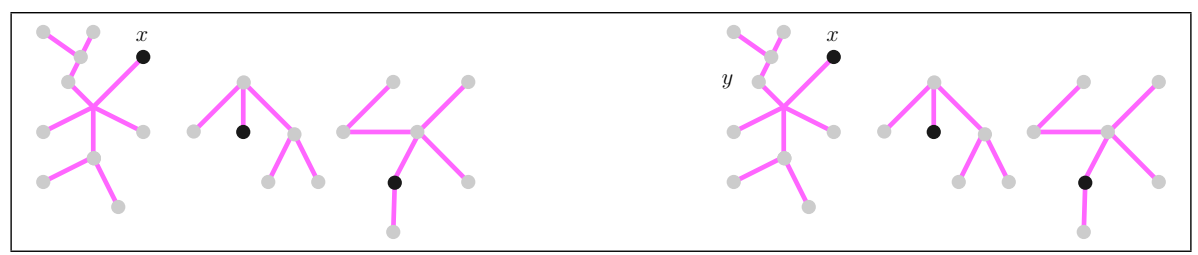

Figure 3: A 3-rooted forest, $x$ is a root (left) and a 3-rooted forest $x$ is a root and $y$ is in the same component (right).

In order to state the matrix notation we need to label the vertices and the edges of the network. So, let $\Gamma=(V, E, c)$ be a network and suppose that $V=\{1, \ldots, n\}$ and $E=\left\{e_{1}, \ldots, e_{m}\right\}$. Assume that each edge of $\Gamma$ is assigned an orientation, which is arbitrary but fixed. Then, $e_{\ell}=(i, j)$, for $\ell=1, \ldots, m$ and $i, j=1, \ldots, n$, where $i$ is the tail and $j$ is the head of $e_{\ell}$. We define the weight of and edge as $w\left(e_{\ell}\right)=c(i, j) \omega(i) \omega(j)$. Then, the weighted incidence matrix $\mathrm{B}_{\omega}=\left(b_{\ell i}\right) \in \mathcal{M}_{m \times n}$ is defined as

$$
b_{\ell i}=\left\{\begin{aligned}
\frac{1}{\omega(i)} & \text { if } i \text { is the head of } e_{\ell}, \\
-\frac{1}{\omega(i)} & \text { if } i \text { is the tail of } e_{\ell}, \\
0 & \text { otherwise, }
\end{aligned}\right.
$$

and we denote by $\mathrm{W}(c, \omega) \in \mathcal{M}_{m}$, the diagonal matrix whose diagonal elements are given by $w\left(e_{\ell}\right)$. Using this notation

$$
\mathrm{L}_{q_{\omega}}=\mathrm{B}_{\omega}^{\top} \mathrm{W}(c, \omega) \mathrm{B}_{\omega} .
$$

Moreover, the matrix associated with $\mathcal{L}_{q}$ is given by

$$
\mathrm{L}_{q}=\mathrm{B}_{\omega}^{\top} \mathrm{W}(c, \omega) \mathrm{B}_{\omega}+\lambda \mathrm{I}
$$

If we denote by $\mathrm{L}_{\hat{q}}^{\lambda, \omega}$, the matrix associated with the Schödinger operator in the host network, Equation (2) can be expressed as

$$
\mathrm{L}_{q}=\mathrm{L}_{\hat{q}}^{\lambda, \omega}(n+1 \mid n+1)
$$

where $\mathrm{L}_{\hat{q}}^{\lambda, \omega}(n+1 \mid n+1)$ is the submatrix of $\mathrm{L}_{\hat{q}}^{\lambda, \omega}$ obtained by deleting the $(n+1)$-th row and column. Moreover, the relation between weighted incidence matrices is the following

$$
\mathrm{B}_{\hat{\omega}}^{\lambda, \omega}=\left[\begin{array}{cc}
\mathrm{B}_{\omega} & 0 \\
\mathrm{D}_{\omega}^{-1} & -\mathrm{j}
\end{array}\right] \text { and } \mathrm{W}^{\lambda, \omega}(c, \omega)=\left[\begin{array}{cc}
\mathrm{W}(c, \omega) & 0 \\
0 & \lambda \mathrm{D}_{\omega}^{2}
\end{array}\right] \text {, }
$$


where $\mathrm{B}_{\hat{\omega}}^{\lambda, \omega} \in \mathcal{M}_{(m+n) \times(n+1)}(\mathbb{R}), \mathrm{W}^{\lambda, \omega}(c, \omega) \in \mathcal{M}_{m+n}(\mathbb{R})$ and $\mathrm{j}$ denotes the all ones vector. Therefore, if $\mathrm{B}_{\hat{\omega}}^{\lambda, \omega}(n+1)$ denotes the submatrix of $\mathrm{B}_{\hat{\omega}}^{\lambda, \omega}$ obtained by deleting the $(n+1)$-th column, we get

$$
\begin{aligned}
\mathrm{L}_{q} & =\mathrm{B}_{\hat{\omega}}^{\lambda, \omega}(n+1)^{\top} \mathrm{W}^{\lambda, \omega}(c, \omega) \mathrm{B}_{\hat{\omega}}^{\lambda, \omega}(n+1) \\
& =\left[\begin{array}{ll}
\mathrm{B}_{\omega}^{\top} & \mathrm{D}_{\omega}^{-1}
\end{array}\right]\left[\begin{array}{cc}
\mathrm{W}(c, \omega) & 0 \\
0 & \lambda \mathrm{D}_{\omega}^{2}
\end{array}\right]\left[\begin{array}{l}
\mathrm{B}_{\omega} \\
\mathrm{D}_{\omega}^{-1}
\end{array}\right]=\mathrm{B}_{\omega}^{T} \mathrm{~W}(c, \omega) \mathrm{B}_{\omega}+\lambda \mathrm{I} .
\end{aligned}
$$

Note, that the above matrices are similar to the ones introduced in [16] in relation with the normalized Laplacian. Notice that with our interpretation these matrices acquire a real meanig.

Observe that if $\hat{\mathcal{F}}$ is a spanning tree on $\Gamma_{\lambda, \omega}$ with vertex $n+1$ as a root and $k$ edges of type $\{n+1, i\}$, the forest generated in $\Gamma$, F, has $k$ connected components and each $i$ belongs to a different component and hence it can be considered as a root of the forest, see Figure 4 .

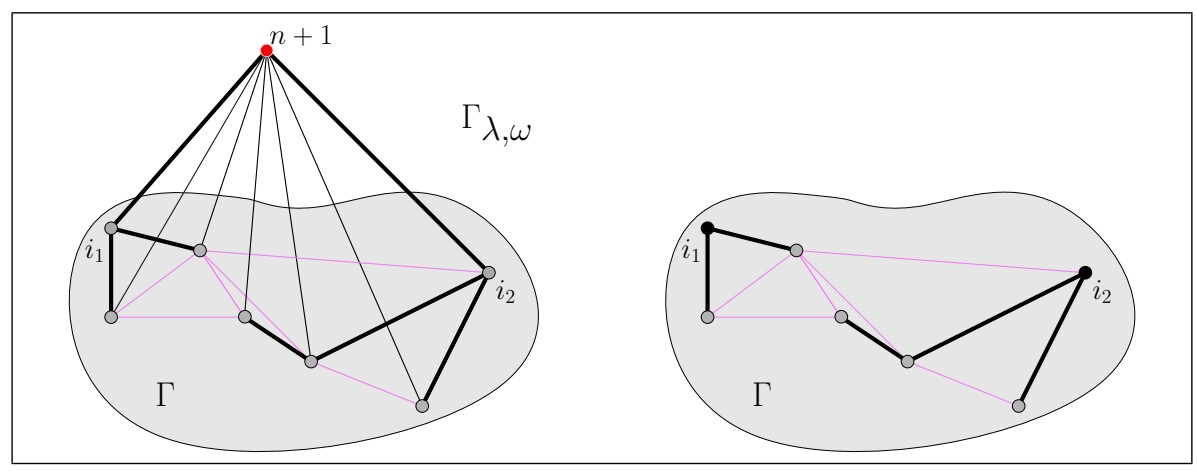

Figure 4: A spanning tree $\hat{\mathcal{F}}$ (left) and the induced 2-rooted forest $\mathcal{F}_{2}$ (right).

With this interpretation of $\hat{\mathcal{F}}$, we can state some generalization of the matrix-tree Theorem. This extension considers not only weights in the edges, as in the previous works, but also weights on the vertices. Moreover, the result applies to general irreducible symmetric $M$-matrices. Although the proof is based on the all minor tree Theorem due to Chaiken, [11], we follow here the clearer terminology introduced in [8].

Call a subset $J=\left\{\left(i_{1}, j_{1}\right), \ldots,\left(i_{\ell}, j_{\ell}\right)\right\} \subset V \times V$ component-disjoint if $i_{p} \neq i_{q}$ and $j_{p} \neq j_{q}$ for every $p \neq q$ and $i_{1}<i_{2}<\cdots<i_{\ell}$; denote $\sum J=\sum_{p=1}^{\ell}\left(i_{p}+j_{p}\right)$. Denote by $\tau_{J}$ the permutation of $\{1,2, \ldots, \ell\}$ defined by the condition $j_{\tau_{J}(1)}<j_{\tau_{J}(2)}<\cdots<j_{\tau_{J}(\ell)}$.

A spanning forest, $\mathcal{F}$, is called $J$-admisible if it has $\ell=|J|$ connected components, and every component contains exactly one vertex from the set $\left\{i_{1}, \ldots, i_{\ell}\right\}$, and exactly one, from $\left\{j_{1}, \ldots, j_{\ell}\right\}$ (the two may coincide if the sets intersect). The vertices $i_{1}, \ldots, i_{\ell}$ will be considered as roots. Denote by $\sigma_{J}^{\mathcal{F}}$ a permutation of $V$ such that $i_{p}$ and $j_{\sigma_{J}^{\mathcal{F}}(p)}$ lie in the same component of $\mathcal{F}$, for every $p=1,2, \ldots, \ell$.

For a matrix $M$ of order $n$ and a component-disjoint set $J$, denote by $M(J)$ the submatrix of $M$ obtained by deletion of the rows $i_{1}, \ldots, i_{\ell}$ and the columns $j_{1}, \ldots, j_{\ell}$. For any permutation $\tau, \epsilon(\tau)$ will denote its sign.

Theorem 2.1. $[8,11]$ For any component-disjoint subset $J \subset V \times V$ it is verified

$$
(-1)^{\sum J} \operatorname{det}\left(\mathrm{L}_{q_{\omega}}\right)(J)=\sum_{\mathcal{F}} \epsilon\left(\tau_{J} \circ \sigma_{J}^{\mathcal{F}}\right) w(\mathcal{F}) \prod_{p=1}^{\ell} \omega\left(i_{p}\right) \omega\left(j_{p}\right),
$$

where the sum is taken over the set of all $J$-admissible forest $\mathcal{F}$ of $\Gamma$ and $\circ$ means the composition of two permutations.

The cases in which $\ell=1,2$ have an special meaning for our pourposes. 
Proposition 2.2. Let $\lambda>0$ and $\omega \in \Omega(V)$. Then, the determinant of the matrix associated with a positive definite Schrödinger operator, $\mathcal{L}_{q}$, is given by

$$
\operatorname{det}\left(\mathrm{L}_{q}\right)=\sum_{k=1}^{n} a_{k} \lambda^{k}
$$

with

$$
a_{k}=\sum_{\mathcal{F} \in \mathcal{F}_{k}} w(\mathcal{F}) \prod_{i_{p} \in r(\mathcal{F})} \omega\left(i_{p}\right)^{2} .
$$

Proof. From Equation (4) and Theorem 2.1 taking $J=\{(n+1, n+1)\}$ and keeping in mind that $\hat{\omega}(n+1)=1$, we get that

$$
\operatorname{det}\left(\mathrm{L}_{q}\right)=\operatorname{det}\left(\mathrm{L}_{\hat{q}}^{\lambda, \omega}(J)\right)=\sum_{\widehat{\mathcal{F}}} \widehat{w}(\hat{\mathcal{F}})
$$

where $\hat{\mathcal{F}}$ is a $J$-admisible forest in $\Gamma_{\lambda, \omega}$ rooted at $n+1$. Notice that in this case the permutations are the identity. Moreover, if $k, k=1, \ldots, n$, is the number of edges of the form $e_{p}=\left\{n+1, i_{p}\right\}, p=1, \ldots, k$, in $\hat{\mathcal{F}}$ and $\mathcal{F}$ is the induced $k$-rooted spanning forest in $\Gamma, k=1, \ldots, n$ with $r(\mathcal{F})=\left\{i_{1}, \ldots, i_{k}\right\}$ as set of roots, we have that

$$
\widehat{w}(\hat{\mathcal{F}})=\frac{\prod_{e_{p}} \lambda \omega\left(i_{p}\right)^{2} \prod_{e \in E(\mathcal{F})} w(e)}{\prod_{j \in V} \omega(j)^{2}}=\lambda^{k} w(\mathcal{F}) \prod_{p=1}^{k} \omega\left(i_{p}\right)^{2} .
$$

Observe that

$$
\operatorname{det}\left(\mathrm{L}_{q}\right)=\operatorname{det}\left(\mathrm{L}_{q_{\omega}}+\lambda \mathrm{I}\right)
$$

and hence $a_{n}$ must equal 1 and $a_{n-1}=\operatorname{tr}\left(\mathrm{L}_{q_{\omega}}\right)=\sum_{x, y \in V} c(x, y) \frac{\omega(x)}{\omega(y)}$. This values can also be deduced from the fact that there is only one forest with $n$ connected components and there are $2 m$ rooted forest with $n-1$ components. Moreover, notice that the above result is also valid for $\lambda=0$, since in this case $\operatorname{det}\left(\mathrm{L}_{q_{\omega}}\right)=0$.

Proposition 2.3. Let $\lambda>0$ and $\omega \in \Omega(V)$. Then, for any $t, s \in V$,

$$
(-1)^{t+s} \operatorname{det}\left(\mathrm{L}_{q}(t \mid s)\right)=\omega(s) \omega(t) \sum_{k=1}^{n} b_{k}(t, s) \lambda^{k-1},
$$

where

$$
b_{k}(t, s)=b_{k}(s, t)=\sum_{\mathcal{F} \in \mathcal{F}_{k, t, s}} w(\mathcal{F}) \prod_{i_{p} \in r^{*}(\mathcal{F})} \omega\left(i_{p}\right)^{2} .
$$

In particular, when $t=s$,

$$
\operatorname{det}\left(\mathrm{L}_{q}(t \mid t)\right)=\sum_{k=1}^{n} \lambda^{k-1} \sum_{\mathcal{F} \in \mathcal{F}_{k, t}} w(\mathcal{F}) \prod_{i_{p} \in r(\mathcal{F})} \omega\left(i_{p}\right)^{2} .
$$

Proof. From Equation (4) and Theorem 2.1 taking $J=\{(t, n+1),(n+1, s)\}$ and keeping in mind that $\hat{\omega}(n+1)=$ 1 , we get that

$$
(-1)^{t+s} \operatorname{det}\left(\mathrm{L}_{q}(t \mid s)\right)=(-1)^{t+s} \operatorname{det}\left(\mathrm{L}_{\hat{q}}^{\lambda, \omega}(J)\right)=\sum_{\hat{\mathcal{F}}} w(\hat{\mathcal{F}}) \omega(t) \omega(s),
$$

where $\hat{\mathcal{F}}$ is a 2 -rooted spanning forest in $\hat{\Gamma}$ rooted at $t$ and $n+1$ and such that $t$ and $s$ belong to the same connected component. Moreover,

$$
\widehat{\omega}(\hat{\mathcal{F}})=\frac{\prod_{e_{p}} \lambda \omega\left(i_{p}\right)^{2} \prod_{e \in \mathcal{F}} w(e)}{\prod_{j \in V} \omega(j)^{2}}=\lambda^{k} \prod_{i_{p}} \omega\left(i_{p}\right)^{2} w(\mathcal{F}),
$$


where $k \in\{0, \ldots, n-1\}$ is the number of edges of the form $e_{p}=\left\{n+1, i_{p}\right\}, p=1, \ldots, k$, and $\mathcal{F}$ is the induced $(k+1)$-rooted spanning forest in $\Gamma, k=0, \ldots, n-1$ with $r(\mathcal{F})=\left\{t, i_{1}, \ldots, i_{k}\right\}$ as set of roots and $t$ and $s$ belong to the same connected component; and hence

$$
(-1)^{t+s} \operatorname{det}\left(\mathrm{L}_{q}(t \mid s)\right)=\omega(s) \omega(t) \sum_{k=0}^{n-1} \lambda^{k} \sum_{\mathcal{F} \in \mathcal{F}_{k+1, t, s}} w(\mathcal{F}) \prod_{i_{p} \in r^{*}(\mathcal{F})} \omega\left(i_{p}\right)^{2},
$$

where $\mathcal{F}$ is a $(k+1)$-rooted spanning forest in $\Gamma$ with $t \in r(\mathcal{F})$ as set of roots and $t, s$ belong to the same connected component. When $t=s$, the formula follows straightforward.

Observe that the above result for the specific case of the normalized Laplacian was obtained by Chung and Zhao in [16, Theorem 3 ], using an intricate proof based on Cauchy-Binet Theorem. Chebotarev et alt., in a serie of papers, also obtained a similar result for the case of weighted (di)graphs for the matrix $L+\lambda l$, $[12,13]$.

The following result represents a full weighted version of the classical matrix tree Theorem, [7, 22]. It can be obtained either directly from Chaiken's Theorem or taking limit when $\lambda$ goes to 0 in the previous result.

Corollary 2.4. Let $\omega \in \Omega(V)$. Then, for any $t, s \in V$,

$$
(-1)^{t+s} \operatorname{det}\left(\mathrm{L}_{q_{\omega}}(t \mid s)\right)=\omega(s) \omega(t) \sum_{\mathcal{F}} w(\mathcal{F}),
$$

where $\mathcal{F}$ runs on the set of spanning trees of $\Gamma$.

\section{The Group inverse of symmetric $M$-matrices}

Using the matrix-tree theorems in the preceding section we can write down a combinatorial expression for the group inverse of any irreducible symmetric $M$-matrix. To do this we consider the associated Schrödinger operator, $\mathcal{L}_{q}$, and then we get its Green function in terms of the weights of rooted spanning forests in $\Gamma$.

Proposition 3.1. Let $\lambda \geq 0$ and $\omega \in \Omega(V)$. Then, for any $t, s \in V$,

$$
G_{q}(t, s)=\omega(s) \omega(t) \frac{\sum_{k=2}^{n} \lambda^{k-2}\left(n b_{k}(t, s)-a_{k}\right)}{n \sum_{k=1}^{n} \lambda^{k-1} a_{k}},
$$

where

$$
b_{k}(t, s)=b_{k}(s, t)=\sum_{\mathcal{F} \in \mathcal{F}_{k, t, s}} w(\mathcal{F}) \prod_{i_{p} \in r^{*}(\mathcal{F})} \omega\left(i_{p}\right)^{2} \text { and } a_{k}=\sum_{\mathcal{F} \in \mathcal{F}_{k}} w(\mathcal{F}) \prod_{i_{p} \in r(\mathcal{F})} \omega\left(i_{p}\right)^{2} .
$$

In particular, $n b_{1}(t, s)=a_{1}$, and hence $b_{1}$ is independent of the variables $t$, s. Moreover, for $\lambda=0$

$$
G_{q_{\omega}}(t, s)=\omega(s) \omega(t) \frac{\left(n b_{2}(t, s)-a_{2}\right)}{n a_{1}} .
$$

Proof. Applying Theorems 2.3 and 2.2 to the adjoint formula for the inverse of a non-singular matrix, we get that

$$
L_{q}^{-1}(t, s)=\omega(s) \omega(t) \frac{\sum_{k=1}^{n} b_{k}(t, s) \lambda^{k-1}}{\lambda \sum_{k=1}^{n} a_{k} \lambda^{k-1}} .
$$

Observe that, $n b_{1}(t, s)=a_{1}$, since

$$
b_{1}(t, s)=\sum_{\mathcal{F} \in \mathcal{F}_{1, t, s}} w(\mathcal{F}) \prod_{i_{p} \in r(\mathcal{F})^{*}} \omega\left(i_{p}\right)^{2}=\sum_{\mathcal{F}} w(\mathcal{F})
$$


and

$$
a_{1}=\sum_{\mathcal{F} \in \mathcal{F}_{1}} w(F) \prod_{i_{p} \in r(\mathcal{F})} \omega\left(i_{p}\right)^{2}=\sum_{\mathcal{F}} \sum_{\substack{\text { spanning } \\ \text { tree }}} w(\mathcal{F}) \sum_{j \in V} \omega^{2}(j)=n \sum_{\substack{\mathcal{F} \text { spanning } \\ \text { tree }}} w(\mathcal{F}) .
$$

Hence, we get that

$$
\frac{G_{q}(t, s)}{\omega(s) \omega(t)}=\frac{L_{q}^{-1}(t, s)}{\omega(s) \omega(t)}-\frac{1}{n \lambda}=\frac{\sum_{k=1}^{n} \lambda^{k-1}\left(n b_{k}(t, s)-a_{k}\right)}{n \lambda \sum_{k=1}^{n} \lambda^{k-1} a_{k}}=\frac{\sum_{k=2}^{n} \lambda^{k-2}\left(n b_{k}(t, s)-a_{k}\right)}{n \sum_{k=1}^{n} \lambda^{k-1} a_{k}} .
$$

The result for $\lambda=0$ follows by taking limit in the above expression.

The following result contains the particular case in which $\omega$ is the constant weight. So, the operator is $\mathcal{L}_{q}=\mathcal{L}+\lambda I$ where $\mathcal{L}$ is the combinatorial Laplacian of a network. This result coincides with the ones obtained in $[13,26,27]$.

Proposition 3.2. Let $\lambda \geq 0$. Then, for any $t, s \in V$,

$$
G_{q}(t, s)=\frac{\sum_{k=2}^{n} \lambda^{k-2}\left(n \sum_{\mathcal{F} \in \mathcal{F}_{k, t, s}} w(\mathcal{F})-\sum_{\mathcal{F} \in \mathcal{F}_{k}} w(\mathcal{F})\right)}{n \sum_{k=1}^{n} \lambda^{k-1} \sum_{\mathcal{F} \in \mathcal{F}_{k}} w(\mathcal{F})} .
$$

In particular, for $\lambda=0$,

$$
G_{q_{\omega}}(t, s)=\frac{n \sum_{\mathcal{F} \in \mathcal{F}_{2, t, s}} w(\mathcal{F})-\sum_{\mathcal{F} \in \mathcal{F}_{2}} w(\mathcal{F})}{n \sum_{\mathcal{F} \in \mathcal{F}_{1}} w(\mathcal{F})} .
$$

For graphs we obtain the expression for the Group inverse of the combinatorial Laplacian $L$ that has been widely considered in the literature, $[13,16]$.

Proposition 3.3. Let $\lambda \geq 0$ and $\mathcal{L}$ the combinatorial Laplacian of a graph. Then, for any $t, s \in V$,

$$
G_{\lambda}(t, s)=\frac{\sum_{k=2}^{n} \lambda^{k-2}\left(n\left|\mathcal{F}_{k, t, s}\right|-\left|\mathcal{F}_{k}\right|\right)}{n \sum_{k=1}^{n} \lambda^{k-1}\left|\mathcal{F}_{k}\right|} .
$$

It means that for $\lambda=0$,

$$
G(t, s)=\frac{\left(n\left|\mathcal{F}_{2, t, s}\right|-\left|\mathcal{F}_{2}\right|\right)}{n\left|\mathcal{F}_{1}\right|} .
$$

In the following example we show how to compute the combinatorial coefficients $a_{k}$ and $b_{k}\left(x_{t}, x_{s}\right)$ in the case of a star on $n$ vertices with center at $x_{1}$ and conductances $c_{i}, i=1, \ldots, n-1$, see Figure 5. In [9, Corollary 5.3] the expression for $G_{\lambda}\left(x_{t}, x_{s}\right)$ was obtained by the authors using different techniques.

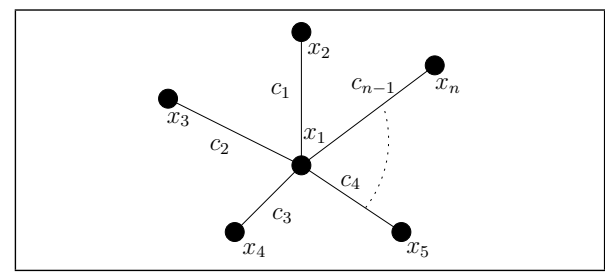

Figure 5: A star on $n$ vertices. 
Proposition 3.4. For a star network with $n$ vertices centered at $x_{1}$ and conductances $c_{i}, i=1, \ldots, n-1$, it is satisfied that

$$
a_{k}=(n-k+1) \sum_{1 \leq i_{1}<\cdots<i_{n-k} \leq n-1} c_{i_{1}} \ldots c_{i_{n-k}}, k=1, \ldots, n .
$$

Moreover, for any $k=2, \ldots, n$, we have

$$
b_{k}\left(x_{1}, x_{1}\right)=\sum_{1 \leq i_{1}<\cdots<i_{n-k} \leq n-1} c_{i_{1}} \ldots c_{i_{n-k}},
$$

for $1<j \leq n$,

$$
b_{k}\left(x_{1}, x_{j}\right)=\sum_{1 \leq i_{1}<\cdots<i_{n-k} \leq n-1} c_{i_{1}} \ldots c_{i_{n-k}}-\sum_{\substack{1 \leq i_{1}<\cdots<i_{n-k} \leq n-1 \\ i_{\ell} \neq j}} c_{i_{1}} \ldots c_{i_{n-k}},
$$

for $1<j \leq n$,

$$
\begin{aligned}
b_{k}\left(x_{j}, x_{j}\right) & =\sum_{1 \leq i_{1}<\cdots<i_{n-k} \leq n-1} c_{i_{1}} \ldots c_{i_{n-k}} \\
& +(n-k) \sum_{\substack{1 \leq i_{1}<\cdots i_{n-k} \leq n-1 \\
i_{\ell} \neq j}} c_{i_{1}} \ldots c_{i_{n-k}},
\end{aligned}
$$

for $1<s, j \leq n, s \neq j$

$$
\begin{aligned}
b_{k}\left(x_{j}, x_{S}\right) & =\sum_{1 \leq i_{1}<\ldots<i_{n-k} \leq n-1} c_{i_{1}} \ldots c_{i_{n-k}}+\sum_{\substack{1 \leq i_{1}<\ldots<i_{n-k} \leq n-1 \\
i_{\ell} \neq j, s}} c_{i_{1}} \ldots c_{i_{n-k}}, \\
& -\sum_{\substack{1 \leq i_{1}<\cdots<i_{n-k} \leq n-1 \\
i_{\ell} \neq j}} c_{i_{1}} \ldots c_{i_{n-k}}-\sum_{\substack{1 \leq i_{1}<\cdots<i_{n-k} \leq n-1 \\
i_{\ell} \neq s}} c_{i_{1}} \ldots c_{i_{n-k}} .
\end{aligned}
$$

To end this section we consider the computation of some small cases that will show the spirit of the results.

The Group inverse for $K_{2}$. Observe that this case encompasses the case of any $2 \times 2$ symmetric $M$-matrix. Consider $\Gamma=(V, E, c)$, where $V=\left\{x_{1}, x_{2}\right\}, E=\left\{e=\left(x_{1}, x_{2}\right)\right\}$ and $c=c\left(x_{1}, x_{2}\right)>0$. Moreover, we consider the weight $\omega_{1}=\omega\left(x_{1}\right), \omega_{2}=\omega\left(x_{2}\right)$, where $\omega_{1}^{2}+\omega_{2}^{2}=2$, the parameter $\lambda \geq 0$ and $q=q_{\omega}+\lambda$. In this case,

$$
\mathrm{L}_{\mathrm{q}}=\left[\begin{array}{cc}
c \frac{\omega_{2}}{\omega_{1}}+\lambda & -c \\
-c & c \frac{\omega_{1}}{\omega_{2}}+\lambda
\end{array}\right]
$$

For $\lambda>0$, we can compute directly the inverse of $L_{q}$, obtaining

$$
\left(\mathrm{L}_{\mathrm{q}}\right)^{-1}=\frac{1}{\lambda\left(\frac{2 c}{\omega_{1} \omega_{2}}+\lambda\right)}\left[\begin{array}{cc}
c \frac{\omega_{1}}{\omega_{2}}+\lambda & c \\
c & c \frac{\omega_{2}}{\omega_{1}}+\lambda
\end{array}\right]=\frac{\omega_{1} \omega_{2}}{\lambda\left(2 c+\lambda \omega_{1} \omega_{2}\right)}\left[\begin{array}{cc}
c \frac{\omega_{1}}{\omega_{2}}+\lambda & c \\
c & c \frac{\omega_{2}}{\omega_{1}}+\lambda
\end{array}\right] .
$$

On the other hand, we get that

$$
\begin{aligned}
& 2 b_{1}\left(x_{i}, x_{j}\right)=a_{1}=2 \frac{c}{\omega_{1} \omega_{2}}, \quad a_{2}=1, \\
& b_{2}\left(x_{1}, x_{1}\right)=\frac{1}{\omega_{1}^{2}}, \quad b_{2}\left(x_{1}, x_{2}\right)=0, \quad b_{2}\left(x_{2}, x_{2}\right)=\frac{1}{\omega_{2}^{2}} .
\end{aligned}
$$

In Figure 6 we can see the existing trees. Therefore, keeping in mind that $a_{1}+\lambda a_{2}=\frac{2 c}{\omega_{1} \omega_{2}}+\lambda$, we get that 


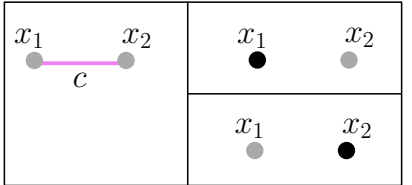

Figure 6: The network $K_{2}$ (left) and the collection of 2-rooted forests for $K_{2}$ (right).

$$
\begin{aligned}
& \left(\mathrm{L}_{q}\right)^{-1}\left(x_{1}, x_{1}\right)=\frac{\omega_{1}^{2}\left(b_{1}\left(x_{1}, x_{1}\right)+\lambda b_{2}\left(x_{1}, x_{1}\right)\right)}{\lambda\left(a_{1}+\lambda a_{2}\right)}=\frac{\frac{c \omega_{1}}{\omega_{2}}+\lambda}{\lambda\left(\frac{2 c}{\omega_{1} \omega_{2}}+\lambda\right)}=\frac{\omega_{1} \omega_{2}}{\lambda\left(2 c+\lambda \omega_{1} \omega_{2}\right)}\left(\frac{c \omega_{1}}{\omega_{2}}+\lambda\right), \\
& \left(\mathrm{L}_{q}\right)^{-1}\left(x_{1}, x_{2}\right)=\frac{\omega_{1} \omega_{2}\left(b_{1}\left(x_{1}, x_{2}\right)+\lambda b_{2}\left(x_{1}, x_{2}\right)\right)}{\lambda\left(a_{1}+\lambda a_{2}\right)}=\frac{c}{\lambda\left(\frac{2 c}{\omega_{1} \omega_{2}}+\lambda\right)}=\frac{\omega_{1} \omega_{2} c}{\lambda\left(2 c+\lambda \omega_{1} \omega_{2}\right)}, \\
& \left(\mathrm{L}_{q}\right)^{-1}\left(x_{2}, x_{2}\right)=\frac{\omega_{2}^{2}\left(b_{1}\left(x_{2}, x_{2}\right)+\lambda b_{2}\left(x_{2}, x_{2}\right)\right)}{\lambda\left(a_{1}+\lambda a_{2}\right)}=\frac{\frac{c \omega_{2}}{\omega_{1}}+\lambda}{\lambda\left(\frac{2 c}{\omega_{1} \omega_{2}}+\lambda\right)}=\frac{\omega_{1} \omega_{2}}{\lambda\left(2 c+\lambda \omega_{1} \omega_{2}\right)}\left(\frac{c \omega_{2}}{\omega_{1}}+\lambda\right) .
\end{aligned}
$$

Moreover,

$$
\begin{aligned}
& \mathrm{G}_{\mathrm{q}}\left(x_{1}, x_{1}\right)=\frac{\omega_{1}^{2}\left(2 b_{2}\left(x_{1}, x_{1}\right)-a_{2}\right)}{2\left(a_{1}+\lambda a_{2}\right)}=\frac{\omega_{2}^{2}}{2\left(\frac{2 c}{\omega_{1} \omega_{2}}+\lambda\right)}=\frac{\omega_{1} \omega_{2}^{3}}{2\left(2 c+\lambda \omega_{1} \omega_{2}\right)}, \\
& \mathrm{G}_{\mathrm{q}}\left(x_{1}, x_{2}\right)=\frac{\omega_{1} \omega_{2}\left(2 b_{2}\left(x_{1}, x_{2}\right)-a_{2}\right)}{2\left(a_{1}+\lambda a_{2}\right)}=\frac{-\omega_{1} \omega_{2}}{2\left(\frac{2 c}{\omega_{1} \omega_{2}}+\lambda\right)}=\frac{-\omega_{1}^{2} \omega_{2}^{2}}{2\left(2 c+\lambda \omega_{1} \omega_{2}\right)}, \\
& \mathrm{G}_{\mathrm{q}}\left(x_{2}, x_{2}\right)=\frac{\omega_{2}^{2}\left(2 b_{2}\left(x_{2}, x_{2}\right)-a_{2}\right)}{2\left(a_{1}+\lambda a_{2}\right)}=\frac{\omega_{1}^{2}}{2\left(\frac{2 c}{\omega_{1} \omega_{2}}+\lambda\right)}=\frac{\omega_{1}^{3} \omega_{2}}{2\left(2 c+\lambda \omega_{1} \omega_{2}\right)} .
\end{aligned}
$$

Finally, for $\lambda=0$

$$
\mathrm{G}_{\mathrm{q}_{\omega}}\left(x_{1}, x_{1}\right)=\frac{\omega_{1} \omega_{2}^{3}}{4 c}, \quad \mathrm{G}_{\mathrm{q}_{\omega}}\left(x_{1}, x_{2}\right)=\frac{-\omega_{1}^{2} \omega_{2}^{2}}{4 c}, \quad \mathrm{G}_{\mathrm{q}_{\omega}}\left(x_{2}, x_{2}\right)=\frac{\omega_{2} \omega_{1}^{3}}{4 c} .
$$

The Group inverse for $K_{3}$. Now, we consider any symmetric $M$-matrix of order 3. For that, Let $\Gamma=$ $(V, E, c)$, where $V=\left\{x_{1}, x_{2}, x_{3}\right\}, E=\left\{e_{1}=\left(x_{1}, x_{2}\right), e_{2}=\left(x_{2}, x_{3}\right), e_{3}=\left(x_{3}, x_{4}\right)\right\}$ and $c_{1}=c\left(x_{1}, x_{2}\right), c_{2}=$ $c\left(x_{2}, x_{3}\right), c_{3}=c\left(x_{3}, x_{1}\right)>0$. Moreover, we consider the weight $\omega_{1}=\omega\left(x_{1}\right), \omega_{2}=\omega\left(x_{2}\right), \omega_{3}=\omega\left(x_{3}\right)$, where $\sum_{k=1}^{3} \omega_{i}^{2}=3, w_{i}=c_{i} \omega_{i} \omega_{i+1}$, the parameter $\lambda \geq 0$ and $q=q_{\omega}+\lambda$. In this case,

$$
\mathrm{L}_{\mathrm{q}}=\left[\begin{array}{ccc}
c_{1} \frac{\omega_{2}}{\omega_{1}}+c_{3} \frac{\omega_{3}}{\omega_{1}}+\lambda & -c_{1} & -c_{3} \\
-c_{1} & c_{1} \frac{\omega_{1}}{\omega_{2}}+c_{2} \frac{\omega_{3}}{\omega_{2}}+\lambda & -c_{2} \\
-c_{3} & -c_{2} & c_{3} \frac{\omega_{1}}{\omega_{3}}+c_{2} \frac{\omega_{2}}{\omega_{3}}+\lambda
\end{array}\right] .
$$

Next, we compute the coefficients $b$ and $a$ involved in the formula of the group inverse. We also represent the trees or forests of any order in Figures 7-9. 


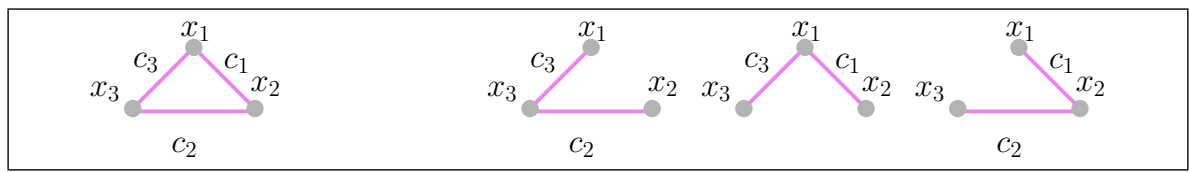

Figure 7: The network $K_{3}$ (left) and the collection of spanning trees for $K_{3}$ (right).

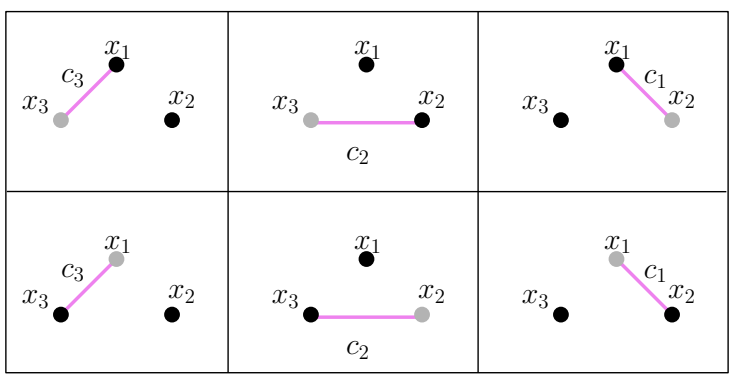

Figure 8: The collection of 2-rooted forests for $K_{3}$.

$$
\begin{aligned}
& a_{1}=3 \prod_{i=1}^{3} c_{i}\left(\frac{1}{w_{1}}+\frac{1}{w_{2}}+\frac{1}{w_{3}}\right), \\
& a_{2}=\prod_{i=1}^{3} c_{i}\left(\frac{\omega_{2}^{2}\left(\omega_{1}^{2}+\omega_{3}^{2}\right)}{w_{1} w_{2}}+\frac{\omega_{1}^{2}\left(\omega_{2}^{2}+\omega_{3}^{2}\right)}{w_{1} w_{3}}+\frac{\omega_{3}^{2}\left(\omega_{1}^{2}+\omega_{2}^{2}\right)}{w_{2} w_{3}}\right), \\
& a_{3}=1=\prod_{i=1}^{3} c_{i} \frac{\omega_{1}^{2} \omega_{2}^{2} \omega_{3}^{2}}{w_{1} w_{2} w_{3}} .
\end{aligned}
$$

To compute coefficients $b_{k}\left(x_{i}, x_{j}\right)$ it is enough to count the $k$-rooted forests displayed at Figures 8 and 9 with $x_{i}$ as a root and $x_{j}$ at the same component as $x_{i}$.

$$
\begin{aligned}
& b_{1}\left(x_{i}, x_{j}\right)=\frac{a_{1}}{3}, \\
& b_{2}\left(x_{1}, x_{1}\right)=\prod_{i=1}^{3} c_{i}\left(\frac{\omega_{2}^{2}}{w_{1} w_{2}}+\frac{\omega_{3}^{2}}{w_{2} w_{3}}+\frac{\left(\omega_{2}^{2}+\omega_{3}^{2}\right)}{w_{1} w_{3}}\right), \\
& b_{2}\left(x_{2}, x_{2}\right)=\prod_{i=1}^{3} c_{i}\left(\frac{\omega_{1}^{2}}{w_{1} w_{3}}+\frac{\omega_{3}^{2}}{w_{2} w_{3}}+\frac{\left(\omega_{1}^{2}+\omega_{3}^{2}\right)}{w_{1} w_{2}}\right), \\
& b_{2}\left(x_{3}, x_{3}\right)=\prod_{i=1}^{3} c_{i}\left(\frac{\omega_{1}^{2}}{w_{1} w_{3}}+\frac{\omega_{2}^{2}}{w_{1} w_{2}}+\frac{\left(\omega_{1}^{2}+\omega_{2}^{2}\right)}{w_{2} w_{3}}\right), \\
& b_{2}\left(x_{1}, x_{2}\right)=\prod_{i=1}^{3} c_{i} \frac{\omega_{3}^{2}}{w_{2} w_{3}}, \quad b_{2}\left(x_{1}, x_{3}\right)=\prod_{i=1}^{3} c_{i} \frac{\omega_{2}^{2}}{w_{1} w_{2}}, \quad b_{2}\left(x_{2}, x_{3}\right)=\prod_{i=1}^{3} c_{i} \frac{\omega_{1}^{2}}{w_{1} w_{3}}, \\
& b_{3}\left(x_{i}, x_{i}\right)=\frac{1}{\omega_{i}^{2}}=\prod_{i=1}^{3} c_{i} \frac{j \neq i}{w_{1} w_{2} w_{3}}, \quad b_{3}\left(x_{i}, x_{j}\right)=0, \text { for all } i \neq j .
\end{aligned}
$$




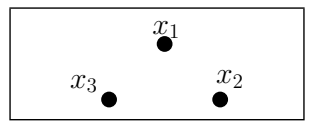

Figure 9: The collection of 3-rooted forests for $K_{3}$.

Applying Theorems 2.3 and 2.2, we get that the inverse of $\mathrm{L}_{q}$ for $K_{3}$ is

$$
\begin{aligned}
& \mathrm{L}_{\mathrm{q}}{ }^{-1}\left(x_{1}, x_{1}\right)=\omega_{1}^{2} \frac{\left(\frac{1}{w_{1}}+\frac{1}{w_{2}}+\frac{1}{w_{3}}\right)+\lambda\left(\frac{\omega_{2}^{2}}{w_{1} w_{2}}+\frac{\omega_{3}^{2}}{w_{2} w_{3}}+\frac{\left(\omega_{2}^{2}+\omega_{3}^{2}\right)}{w_{1} w_{3}}\right)+\lambda^{2} \frac{\omega_{2}^{2} \omega_{3}^{2}}{w_{1} w_{2} w_{3}}}{3 \lambda\left(\frac{1}{w_{1}}+\frac{1}{w_{2}}+\frac{1}{w_{3}}\right)+\lambda^{2}\left(\frac{\omega_{2}^{2}\left(\omega_{1}^{2}+\omega_{3}^{2}\right)}{w_{1} w_{2}}+\frac{\omega_{1}^{2}\left(\omega_{2}^{2}+\omega_{3}^{2}\right)}{w_{1} w_{3}}+\frac{\omega_{3}^{2}\left(\omega_{1}^{2}+\omega_{2}^{2}\right)}{w_{2} w_{3}}\right)+\lambda^{3} \frac{\omega_{1}^{2} \omega_{2}^{2} \omega_{3}^{2}}{w_{1} w_{2} w_{3}},} \\
& \mathrm{~L}_{\mathrm{q}}^{-1}\left(x_{2}, x_{2}\right)=\omega_{2}^{2} \frac{\left(\frac{1}{w_{1}}+\frac{1}{w_{2}}+\frac{1}{w_{3}}\right)+\lambda\left(\frac{\omega_{1}^{2}}{w_{1} w_{3}}+\frac{\omega_{3}^{2}}{w_{2} w_{3}}+\frac{\left(\omega_{1}^{2}+\omega_{3}^{2}\right)}{w_{1} w_{2}}\right)+\lambda^{2} \frac{\omega_{1}^{2} \omega_{3}^{2}}{w_{1} w_{2} w_{3}}}{3 \lambda\left(\frac{1}{w_{1}}+\frac{1}{w_{2}}+\frac{1}{w_{3}}\right)+\lambda^{2}\left(\frac{\omega_{2}^{2}\left(\omega_{1}^{2}+\omega_{3}^{2}\right)}{w_{1} w_{2}}+\frac{\omega_{1}^{2}\left(\omega_{2}^{2}+\omega_{3}^{2}\right)}{w_{1} w_{3}}+\frac{\omega_{3}^{2}\left(\omega_{1}^{2}+\omega_{2}^{2}\right)}{w_{2} w_{3}}\right)+\lambda^{3} \frac{\omega_{1}^{2} \omega_{2}^{2} \omega_{3}^{2}}{w_{1} w_{2} w_{3}}}, \\
& \mathrm{~L}_{\mathrm{q}}^{-1}\left(x_{3}, x_{3}\right)=\omega_{3}^{2} \frac{\left(\frac{1}{w_{1}}+\frac{1}{w_{2}}+\frac{1}{w_{3}}\right)+\lambda\left(\frac{\omega_{1}^{2}}{w_{1} w_{3}}+\frac{\omega_{2}^{2}}{w_{1} w_{2}}+\frac{\left(\omega_{1}^{2}+\omega_{2}^{2}\right)}{w_{2} w_{3}}\right)+\lambda^{2} \frac{\omega_{1}^{2} \omega_{2}^{2}}{w_{1} w_{2} w_{3}}}{3 \lambda\left(\frac{1}{w_{1}}+\frac{1}{w_{2}}+\frac{1}{w_{3}}\right)+\lambda^{2}\left(\frac{\omega_{2}^{2}\left(\omega_{1}^{2}+\omega_{3}^{2}\right)}{w_{1} w_{2}}+\frac{\omega_{1}^{2}\left(\omega_{2}^{2}+\omega_{3}^{2}\right)}{w_{1} w_{3}}+\frac{\omega_{3}^{2}\left(\omega_{1}^{2}+\omega_{2}^{2}\right)}{w_{2} w_{3}}\right)+\lambda^{3} \frac{\omega_{1}^{2} \omega_{2}^{2} \omega_{3}^{2}}{w_{1} w_{2} w_{3}}}, \\
& \mathrm{Lq}^{-1}\left(x_{1}, x_{2}\right)=\omega_{1} \omega_{2} \frac{\left(\frac{1}{w_{1}}+\frac{1}{w_{2}}+\frac{1}{w_{3}}\right)+\lambda \frac{\omega_{3}^{2}}{w_{2} w_{3}}}{3 \lambda\left(\frac{1}{w_{1}}+\frac{1}{w_{2}}+\frac{1}{w_{3}}\right)+\lambda^{2}\left(\frac{\omega_{2}^{2}\left(\omega_{1}^{2}+\omega_{3}^{2}\right)}{w_{1} w_{2}}+\frac{\omega_{1}^{2}\left(\omega_{2}^{2}+\omega_{3}^{2}\right)}{w_{1} w_{3}}+\frac{\omega_{3}^{2}\left(\omega_{1}^{2}+\omega_{2}^{2}\right)}{w_{2} w_{3}}\right)+\lambda^{3} \frac{\omega_{1}^{2} \omega_{2}^{2} \omega_{3}^{2}}{w_{1} w_{2} w_{3}}}, \\
& \mathrm{~L}^{-1}\left(x_{1}, x_{3}\right)=\omega_{1} \omega_{3} \frac{\left(\frac{1}{w_{1}}+\frac{1}{w_{2}}+\frac{1}{w_{3}}\right)+\lambda \frac{\omega_{2}^{2}}{w_{1} w_{2}}}{3 \lambda\left(\frac{1}{w_{1}}+\frac{1}{w_{2}}+\frac{1}{w_{3}}\right)+\lambda^{2}\left(\frac{\omega_{2}^{2}\left(\omega_{1}^{2}+\omega_{3}^{2}\right)}{w_{1} w_{2}}+\frac{\omega_{1}^{2}\left(\omega_{2}^{2}+\omega_{3}^{2}\right)}{w_{1} w_{3}}+\frac{\omega_{3}^{2}\left(\omega_{1}^{2}+\omega_{2}^{2}\right)}{w_{2} w_{3}}\right)+\lambda^{3} \frac{\omega_{1}^{2} \omega_{2}^{2} \omega_{3}^{2}}{w_{1} w_{2} w_{3}}}, \\
& \mathrm{Lq}^{-1}\left(x_{2}, x_{3}\right)=\omega_{2} \omega_{3} \frac{\left(\frac{1}{w_{1}}+\frac{1}{w_{2}}+\frac{1}{w_{3}}\right)+\lambda \frac{\omega_{1}^{2}}{w_{1} w_{3}}}{3 \lambda\left(\frac{1}{w_{1}}+\frac{1}{w_{2}}+\frac{1}{w_{3}}\right)+\lambda^{2}\left(\frac{\omega_{2}^{2}\left(\omega_{1}^{2}+\omega_{3}^{2}\right)}{w_{1} w_{2}}+\frac{\omega_{1}^{2}\left(\omega_{2}^{2}+\omega_{3}^{2}\right)}{w_{1} w_{3}}+\frac{\omega_{3}^{2}\left(\omega_{1}^{2}+\omega_{2}^{2}\right)}{w_{2} w_{3}}\right)+\lambda^{3} \frac{\omega_{1}^{2} \omega_{2}^{2} \omega_{3}^{2}}{w_{1} w_{2} w_{3}}} .
\end{aligned}
$$


Moreover, from Proposition 4.1 we get that the expression for the Green function for $K_{3}$ and $\lambda \geq 0$ is

$$
\begin{aligned}
& 3 G_{q}\left(x_{1}, x_{1}\right)=\frac{\omega_{1}^{2}\left(\frac{\omega_{2}^{4}}{w_{1} w_{2}}+\frac{\omega_{3}^{4}}{w_{2} w_{3}}+\frac{\left(\omega_{2}^{2}+\omega_{3}^{2}\right)^{2}}{w_{1} w_{3}}+\lambda \frac{\omega_{2}^{2} \omega_{3}^{2}\left(\omega_{2}^{2}+\omega_{3}^{2}\right)}{w_{1} w_{2} w_{3}}\right)}{3\left(\frac{1}{w_{1}}+\frac{1}{w_{2}}+\frac{1}{w_{3}}\right)+\lambda\left(\frac{\omega_{2}^{2}\left(\omega_{1}^{2}+\omega_{3}^{2}\right)}{w_{1} w_{2}}+\frac{\omega_{1}^{2}\left(\omega_{2}^{2}+\omega_{3}^{2}\right)}{w_{1} w_{3}}+\frac{\omega_{3}^{2}\left(\omega_{1}^{2}+\omega_{2}^{2}\right)}{w_{2} w_{3}}\right)+\lambda^{2} \frac{\omega_{1}^{2} \omega_{2}^{2} \omega_{3}^{2}}{w_{1} w_{2} w_{3}}}, \\
& 3 G_{q}\left(x_{2}, x_{2}\right)=\frac{\omega_{2}^{2}\left(\frac{\omega_{1}^{4}}{w_{1} w_{3}}+\frac{\omega_{3}^{4}}{w_{2} w_{3}}+\frac{\left(\omega_{1}^{2}+\omega_{3}^{2}\right)^{2}}{w_{1} w_{2}}+\lambda \frac{\omega_{1}^{2} \omega_{3}^{2}\left(\omega_{1}^{2}+\omega_{3}^{2}\right)}{w_{1} w_{2} w_{3}}\right)}{3\left(\frac{1}{w_{1}}+\frac{1}{w_{2}}+\frac{1}{w_{3}}\right)+\lambda\left(\frac{\omega_{2}^{2}\left(\omega_{1}^{2}+\omega_{3}^{2}\right)}{w_{1} w_{2}}+\frac{\omega_{1}^{2}\left(\omega_{2}^{2}+\omega_{3}^{2}\right)}{w_{1} w_{3}}+\frac{\omega_{3}^{2}\left(\omega_{1}^{2}+\omega_{2}^{2}\right)}{w_{2} w_{3}}\right)+\lambda^{2} \frac{\omega_{1}^{2} \omega_{2}^{2} \omega_{3}^{2}}{w_{1} w_{2} w_{3}}}, \\
& 3 G_{q}\left(x_{3}, x_{3}\right)=\frac{\omega_{3}^{2}\left(\frac{\omega_{1}^{4}}{w_{1} w_{3}}+\frac{\omega_{2}^{4}}{w_{1} w_{2}}+\frac{\left(\omega_{1}^{2}+\omega_{2}^{2}\right)^{2}}{w_{2} w_{3}}+\lambda \frac{\omega_{1}^{2} \omega_{2}^{2}\left(\omega_{1}^{2}+\omega_{2}^{2}\right)}{w_{1} w_{2} w_{3}}\right)}{3\left(\frac{1}{w_{1}}+\frac{1}{w_{2}}+\frac{1}{w_{3}}\right)+\lambda\left(\frac{\omega_{2}^{2}\left(\omega_{1}^{2}+\omega_{3}^{2}\right)}{w_{1} w_{2}}+\frac{\omega_{1}^{2}\left(\omega_{2}^{2}+\omega_{3}^{2}\right)}{w_{1} w_{3}}+\frac{\omega_{3}^{2}\left(\omega_{1}^{2}+\omega_{2}^{2}\right)}{w_{2} w_{3}}\right)+\lambda^{2} \frac{\omega_{1}^{2} \omega_{2}^{2} \omega_{3}^{2}}{w_{1} w_{2} w_{3}}}, \\
& 3 G_{q}\left(x_{1}, x_{2}\right)=\frac{\omega_{1} \omega_{2}\left(\frac{\omega_{3}^{4}}{w_{2} w_{3}}-\frac{\omega_{2}^{2}\left(\omega_{1}^{2}+\omega_{3}^{2}\right)}{w_{1} w_{2}}-\frac{\omega_{1}^{2}\left(\omega_{2}^{2}+\omega_{3}^{2}\right)}{w_{1} w_{3}}-\lambda \frac{\omega_{1}^{2} \omega_{2}^{2} \omega_{3}^{2}}{w_{1} w_{2} w_{3}}\right)}{3\left(\frac{1}{w_{1}}+\frac{1}{w_{2}}+\frac{1}{w_{3}}\right)+\lambda\left(\frac{\omega_{2}^{2}\left(\omega_{1}^{2}+\omega_{3}^{2}\right)}{w_{1} w_{2}}+\frac{\omega_{1}^{2}\left(\omega_{2}^{2}+\omega_{3}^{2}\right)}{w_{1} w_{3}}+\frac{\omega_{3}^{2}\left(\omega_{1}^{2}+\omega_{2}^{2}\right)}{w_{2} w_{3}}\right)+\lambda^{2} \frac{\omega_{1}^{2} \omega_{2}^{2} \omega_{3}^{2}}{w_{1} w_{2} w_{3}}}, \\
& 3 G_{q}\left(x_{1}, x_{3}\right)=\frac{\omega_{1} \omega_{3}\left(\frac{\omega_{2}^{4}}{w_{1} w_{2}}-\frac{\omega_{1}^{2}\left(\omega_{2}^{2}+\omega_{3}^{2}\right)}{w_{1} w_{3}}-\frac{\omega_{3}^{2}\left(\omega_{1}^{2}+\omega_{2}^{2}\right)}{w_{2} w_{3}}-\lambda \frac{\omega_{1}^{2} \omega_{2}^{2} \omega_{3}^{2}}{w_{1} w_{2} w_{3}}\right)}{3\left(\frac{1}{w_{1}}+\frac{1}{w_{2}}+\frac{1}{w_{3}}\right)+\lambda\left(\frac{\omega_{2}^{2}\left(\omega_{1}^{2}+\omega_{3}^{2}\right)}{w_{1} w_{2}}+\frac{\omega_{1}^{2}\left(\omega_{2}^{2}+\omega_{3}^{2}\right)}{w_{1} w_{3}}+\frac{\omega_{3}^{2}\left(\omega_{1}^{2}+\omega_{2}^{2}\right)}{w_{2} w_{3}}\right)+\lambda^{2} \frac{\omega_{1}^{2} \omega_{2}^{2} \omega_{3}^{2}}{w_{1} w_{2} w_{3}}}, \\
& 3 G_{q}\left(x_{2}, x_{3}\right)=\frac{\omega_{1} \omega_{2}\left(\frac{\omega_{1}^{4}}{w_{1} w_{3}}-\frac{\omega_{2}^{2}\left(\omega_{1}^{2}+\omega_{3}^{2}\right)}{w_{1} w_{2}}-\frac{\omega_{3}^{2}\left(\omega_{1}^{2}+\omega_{2}^{2}\right)}{w_{2} w_{3}}-\lambda \frac{\omega_{1}^{2} \omega_{2}^{2} \omega_{3}^{2}}{w_{1} w_{2} w_{3}}\right)}{3\left(\frac{1}{w_{1}}+\frac{1}{w_{2}}+\frac{1}{w_{3}}\right)+\lambda\left(\frac{\omega_{2}^{2}\left(\omega_{1}^{2}+\omega_{3}^{2}\right)}{w_{1} w_{2}}+\frac{\omega_{1}^{2}\left(\omega_{2}^{2}+\omega_{3}^{2}\right)}{w_{1} w_{3}}+\frac{\omega_{3}^{2}\left(\omega_{1}^{2}+\omega_{2}^{2}\right)}{w_{2} w_{3}}\right)+\lambda^{2} \frac{\omega_{1}^{2} \omega_{2}^{2} \omega_{3}^{2}}{w_{1} w_{2} w_{3}}} .
\end{aligned}
$$

In particular, for $\lambda=0$, we get that

$$
\begin{aligned}
& \frac{G_{q}\left(x_{1}, x_{1}\right)}{\omega_{1}^{2}}=\frac{\frac{\omega_{2}^{4}}{w_{1} w_{2}}+\frac{\omega_{3}^{4}}{w_{2} w_{3}}+\frac{\left(\omega_{2}^{2}+\omega_{3}^{2}\right)^{2}}{w_{1} w_{3}}}{9\left(\frac{1}{w_{1}}+\frac{1}{w_{2}}+\frac{1}{w_{3}}\right)}, \quad \frac{G_{q}\left(x_{1}, x_{2}\right)}{\omega_{1} \omega_{2}}=\frac{\frac{\omega_{3}^{4}}{w_{2} w_{3}}-\frac{\omega_{2}^{2}\left(\omega_{1}^{2}+\omega_{3}^{2}\right)}{w_{1} w_{2}}-\frac{\omega_{1}^{2}\left(\omega_{2}^{2}+\omega_{3}^{2}\right)}{w_{1} w_{3}}}{9\left(\frac{1}{w_{1}}+\frac{1}{w_{2}}+\frac{1}{w_{3}}\right)}, \\
& \frac{G_{q}\left(x_{2}, x_{2}\right)}{\omega_{2}^{2}}=\frac{\frac{\omega_{1}^{4}}{w_{1} w_{3}}+\frac{\omega_{3}^{4}}{w_{2} w_{3}}+\frac{\left(\omega_{1}^{2}+\omega_{3}^{2}\right)^{2}}{w_{1} w_{2}}}{9\left(\frac{1}{w_{1}}+\frac{1}{w_{2}}+\frac{1}{w_{3}}\right)}, \quad \frac{G_{q}\left(x_{1}, x_{3}\right)}{\omega_{1} \omega_{3}}=\frac{\frac{\omega_{2}^{4}}{w_{1} w_{2}}-\frac{\omega_{1}^{2}\left(\omega_{2}^{2}+\omega_{3}^{2}\right)}{w_{1} w_{3}}-\frac{\omega_{3}^{2}\left(\omega_{1}^{2}+\omega_{2}^{2}\right)}{w_{2} w_{3}}}{9\left(\frac{1}{w_{1}}+\frac{1}{w_{2}}+\frac{1}{w_{3}}\right)}, \\
& \frac{G_{q}\left(x_{3}, x_{3}\right)}{\omega_{3}^{2}}=\frac{\frac{\omega_{1}^{4}}{w_{1} w_{3}}+\frac{\omega_{2}^{4}}{w_{1} w_{2}}+\frac{\left(\omega_{1}^{2}+\omega_{2}^{2}\right)^{2}}{w_{2} w_{3}}}{9\left(\frac{1}{w_{1}}+\frac{1}{w_{2}}+\frac{1}{w_{3}}\right)}, \quad \frac{G_{q}\left(x_{2}, x_{3}\right)}{\omega_{2} \omega_{3}}=\frac{\frac{\omega_{1}^{4}}{w_{1} w_{3}}-\frac{\omega_{2}^{2}\left(\omega_{1}^{2}+\omega_{3}^{2}\right)}{w_{1} w_{2}}-\frac{\omega_{3}^{2}\left(\omega_{1}^{2}+\omega_{2}^{2}\right)}{w_{2} w_{3}}}{9\left(\frac{1}{w_{1}}+\frac{1}{w_{2}}+\frac{1}{w_{3}}\right)} .
\end{aligned}
$$




\section{The group inverse for trees}

Consider now a weighted tree with constant weights on the vertex set, $\mathcal{T}$. For any $k \geq 1$ and any set of edges $\varepsilon_{k} \subset E(\mathcal{T})$ satisfying $\left|\varepsilon_{k}\right|=k-1$, we let $\mathcal{T} \backslash \varepsilon_{k}$ denote the graph obtained from $\mathcal{T}$ by deleting the edges of $\varepsilon_{k}$. Observe that, $\mathcal{T} \backslash \varepsilon_{k}$ is a forest that has $k$ connected components, that will be denoted by $C_{i}\left(\varepsilon_{k}\right), i=1, \ldots, k$. For each set $\varepsilon_{k} \in E(\mathcal{T})$ and a vertex $t \in \mathcal{T}$, we denote $C_{i}^{t}\left(\mathcal{E}_{k}\right), i=1, \ldots, k-1$, the set of vertices in the $i$-th connected component of $\mathcal{T} \backslash \varepsilon_{k}$ that does not contain vertex $t$. Moreover, for any pair of vertices $t, s \in V(\mathcal{T})$, we denote by $\varepsilon_{k}^{t \rightarrow s} \subset E(\mathcal{T})$ a set of $k-1$ edges that are not in the unique path from $t$ to $s$ in $\mathcal{T}, \mathcal{P}_{t, s}$. Finally, denote by $w\left(\varepsilon_{k}\right)=\prod_{e \in \varepsilon_{k}} c(e)$.

Corollary 4.1. Let $\lambda \geq 0$. Then, for any $t, s \in \mathcal{T}$

$$
G_{\lambda}(t, s)=\frac{\sum_{k=2}^{n} \lambda^{k-2}\left(n \sum_{\varepsilon_{k}^{t \rightarrow s}} \frac{\prod_{i=1}^{k-1}\left|C_{i}^{t}\left(\varepsilon_{k}^{t \rightarrow s}\right)\right|}{w\left(\mathcal{E}_{k}^{t \rightarrow s}\right)}-\sum_{\mathcal{E}_{k}} \frac{\prod_{i=1}^{k}\left|C_{i}\left(\mathcal{E}_{k}\right)\right|}{w\left(\mathcal{E}_{k}\right)}\right)}{n \sum_{k=1}^{n} \lambda^{k-1} \sum_{\mathcal{E}_{k}} \frac{\prod_{i=1}^{k}\left|C_{i}\left(\varepsilon_{k}\right)\right|}{w\left(\mathcal{E}_{k}\right)}} .
$$

In particular, for $\lambda=0$ we have that

$$
G(t, s)=\frac{1}{n^{2}} \sum_{e \in E(\mathcal{T})} \frac{\left|C_{1}^{t}(e)\right|^{2}}{c(e)}-\frac{1}{n} \sum_{e \in \mathcal{P}_{t, s}} \frac{\left|C_{1}^{t}(e)\right|}{c(e)} .
$$

Proof. It is enough to observe that for any $t, s \in V(\mathcal{T})$, and any choice of edges $\varepsilon_{k}^{t \rightarrow s}=\left\{e_{1}, \ldots, e_{k-1}\right\}$, there exist $\prod_{i=1}^{k-1}\left|C_{i}^{t}\left(\varepsilon_{k}^{t \rightarrow s}\right)\right|$ different $k$-rooted forest with $t$ as a root and $\prod_{i=1}^{k}\left|C_{i}\left(\mathcal{E}_{k}\right)\right|$ different $k$-rooted forest whose weights are given by

$$
w\left(\mathcal{T} \backslash \mathcal{E}_{k}^{t \rightarrow s}\right)=\frac{\prod_{e \in E(\mathcal{T})} c(e)}{w\left(\mathcal{E}_{k}^{t \rightarrow s}\right)} \text { and } w\left(\mathcal{T} \backslash \mathcal{E}_{k}\right)=\frac{\prod_{e \in E(\mathcal{T})} c(e)}{w\left(\mathcal{E}_{k}\right)}
$$

and hence the results follow by applying Proposition 4.1.

Observe that the result in the above Corollary for $\lambda=0$, coincides with the one obtained in [23, Corollary 7.2.6], see also [24, Lemma 2.14].

In particular, if we consider a path on $n$ vertices and keeping in mind that $\left|C_{1}^{t}\left(e_{k}\right)\right|=k$ for any $e_{k}$ whose initial vertex is $k \leq t-1$ and $\left|C_{1}^{t}\left(e_{k}\right)\right|=n-k$ for any $e_{k}$ whose initial vertex is $k \geq t$, we recover the expression for the Green function obtained by the authors in [5]

$$
G(t, s)=\frac{1}{n^{2}}\left(\sum_{k=1}^{\min \{t, s\}-1} \frac{k^{2}}{c\left(e_{k}\right)}+\sum_{k=\min \{t, s\}}^{n-1} \frac{(n-k)^{2}}{c\left(e_{k}\right)}\right)-\frac{1}{n} \sum_{k=\min \{t, s\}}^{\max \{t, s\}} \frac{(n-k)}{c\left(e_{k}\right)} .
$$

Proposition 4.2. Let $\lambda>0$ and $\mathcal{L}$ the combinatorial Laplacian of a weighted tree, $\mathcal{T}$. Then, for any $t, s \in V(\mathcal{T})$,

$$
L_{\lambda}^{-1}(t, s)=\frac{1+\sum_{k=2}^{n} \lambda^{k-1} \sum_{\mathcal{E}_{k}^{t \rightarrow s}} w^{-1}\left(\mathcal{E}_{k}^{t \rightarrow s}\right) \prod_{i=1}^{k-1}\left|C_{i}^{t}\left(\mathcal{E}_{k}^{t \rightarrow s}\right)\right|}{\lambda\left(n+\sum_{k=2}^{n} \lambda^{k-1} \sum_{\varepsilon_{k}} w^{-1}\left(\mathcal{E}_{k}\right) \prod_{i=1}^{k}\left|C_{i}\left(\varepsilon_{k}\right)\right|\right)} .
$$

In particular,

$$
L_{\lambda}^{-1}(t, t)=\frac{1+\sum_{k=2}^{n} \lambda^{k-1} \sum_{\mathcal{E}_{k}} w^{-1}\left(\mathcal{E}_{k}\right) \prod_{i=1}^{k-1}\left|C_{i}^{t}\left(\mathcal{E}_{k}\right)\right|}{\left.\lambda\left(n+\sum_{k=2}^{n} \lambda^{k-1} \sum_{\mathcal{E}_{k}} w^{-1}\left(\mathcal{E}_{k}\right) \prod_{i=1}^{k}\left|C_{i}\left(\mathcal{E}_{k}\right)\right|\right)\right)} .
$$


For the case of a path with constant conductances the authors obtained in [3] the inverse of $L_{\lambda}$ by using difference equation techniques and comparing both expressions we can state the following in some sense surprising identities.

Corollary 4.3. Let $\lambda>0$ with the above notations, we get that

$$
n+\sum_{k=2}^{n} \lambda^{k-1} \sum_{\varepsilon_{k}} \prod_{i=1}^{k}\left|C_{i}\left(\varepsilon_{k}\right)\right|=U_{n-1}\left(1+\frac{\lambda}{2}\right)
$$

and for $t \leq s$

$$
1+\sum_{k=2}^{n} \lambda^{k-1} \sum_{\varepsilon_{k}^{t \rightarrow s}} \prod_{i=1}^{k-1}\left|C_{i}^{t}\left(\varepsilon_{k}^{t \rightarrow s}\right)\right|=V_{t-1}\left(1+\frac{\lambda}{2}\right) V_{n-s}\left(1+\frac{\lambda}{2}\right),
$$

where $U_{n}(x)$ and $V_{n}(x)$ are the second and third kind Chebyshev polynomials.

It is notable that different values of the parameter $\lambda$ enable us to give more or less importance to the length of the different walks between vertices, $[10,15]$. Moreover, we can obtain some relations between combinatorial numbers and the number of spanning rooted trees. First, let us define the Horadam numbers $H_{n}(r, s)$ as the solution of the following three term recurrence sequence

$$
H_{n+2}(r, s)=r H_{n+1}(r, s)+s H_{n}(r, s), H_{0}(r, s)=0, H_{1}(r, s)=1, r, s \in \mathbb{Z} .
$$

See [19] for properties of Horadam numbers. In particular, it is well known that $H_{n}(1,1)=F_{n}$, the $n$-th Fibonacci number; $H_{n}(2,1)=P_{n}$, the $n$-th Pell number; $H_{n}(1,2)=J_{n}$, the $n$-th Jacobsthal number and $H_{n}(2,-1)=n$. Moreover, if we allow $r, s \in \mathbb{R}$, we can obtain the relation between Horadam solutions and the second kind Chebyshev polynomials: If $s<0, H_{n}(r, s)=(\sqrt{-s})^{n-1} U_{n-1}\left(\frac{r}{2 \sqrt{-s}}\right)$ and if $s>0$, $H_{2 n}(r, s)=r s^{n-1} U_{n-1}\left(1+\frac{r^{2}}{2 s}\right)$.

Corollary 4.4. For a path on $n$ vertices whose conductances equal 1 and using the above notations, it is satisfied that

$$
n+\sum_{k=2}^{n} \lambda^{k-1} \sum_{\mathcal{E}_{k}} \prod_{i=1}^{k}\left|C_{i}\left(\mathcal{E}_{k}\right)\right|=\frac{1}{\lambda^{n}} H_{2 n}(\lambda, \lambda)=\frac{1}{\sqrt{\lambda}} H_{2 n}(\sqrt{\lambda}, 1)=\lambda^{n-1} H_{2 n}\left(1, \lambda^{-1}\right) .
$$

In particular for $\lambda=1,4$ and $\frac{1}{2}$, we get

$$
\begin{aligned}
n+\sum_{k=2}^{n} \sum_{\varepsilon_{k}} \prod_{i=1}^{k}\left|C_{i}\left(\varepsilon_{k}\right)\right| & =F_{2 n}, \\
n+\sum_{k=2}^{n} 4^{k-1} \sum_{\varepsilon_{k}} \prod_{i=1}^{k}\left|C_{i}\left(\varepsilon_{k}\right)\right| & =\frac{1}{2} P_{2 n}, \\
n+\sum_{k=2}^{n} \frac{1}{2^{k-1}} \sum_{\mathcal{E}_{k}} \prod_{i=1}^{k}\left|C_{i}\left(\varepsilon_{k}\right)\right| & =\frac{1}{2^{n-1}} J_{2 n} .
\end{aligned}
$$

Observe that the case $\lambda=1$ gives that $F_{2 n}$ is the total number of spanning rooted forests of the path, see [14] for a different proof of this result. On the other hand, $P_{2 n}$ represents the number of rooted spanning forest taking into account a weight associated with the number of components of the forest, so the more components the greater the weight. In contrast, for the numbers $J_{2 n}$ the less components the greater the weight.

\section{The Group inverse for $C_{4}$}

In this section we consider a cycle of order 4, and hence we look for the inverse or the Green function of some especial class of symmetric $M$-matrix of order 4. Let $\Gamma=(V, E, c)$, where $V=\left\{x_{1}, x_{2}, x_{3}, x_{4}\right\}, E=\left\{e_{1}=\right.$ 
$\left.\left(x_{1}, x_{2}\right), e_{2}=\left(x_{2}, x_{3}\right), e_{3}=\left(x_{3}, x_{4}\right), e_{4}=\left(x_{4}, x_{1}\right)\right\}$ and $c_{1}=c\left(x_{1}, x_{2}\right), c_{2}=c\left(x_{2}, x_{3}\right), c_{3}=c\left(x_{3}, x_{4}\right), c_{4}=$ $c\left(x_{4}, x_{1}\right)>0$. Moreover, we consider the weight $\omega_{1}=\omega\left(x_{1}\right), \omega_{2}=\omega\left(x_{2}\right), \omega_{3}=\omega\left(x_{3}\right), \omega_{4}=\omega\left(x_{4}\right)$, where $\sum_{k=1}^{4} \omega_{i}^{2}=4, w_{i}=c_{i} \omega_{i} \omega_{i+1}$, the parameter $\lambda \geq 0$ and $\mathbf{q}=q_{\omega}+\lambda$. In this case,

$$
\mathrm{L}_{\mathrm{q}}=\left[\begin{array}{cccc}
c_{1} \frac{\omega_{2}}{\omega_{1}}+c_{4} \frac{\omega_{4}}{\omega_{1}}+\lambda & -c_{1} & 0 & -c_{4} \\
-c_{1} & c_{1} \frac{\omega_{1}}{\omega_{2}}+c_{2} \frac{\omega_{3}}{\omega_{2}}+\lambda & -c_{2} & 0 \\
0 & -c_{2} & c_{2} \frac{\omega_{2}}{\omega_{3}}+c_{3} \frac{\omega_{4}}{\omega_{3}}+\lambda & -c_{3} \\
-c_{4} & 0 & -c_{3} & c_{4} \frac{\omega_{1}}{\omega_{4}}+c_{3} \frac{\omega_{3}}{\omega_{4}}+\lambda
\end{array}\right] .
$$

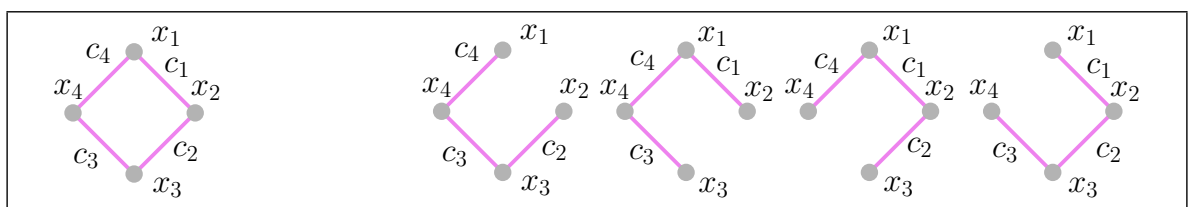

Figure 10: The network $C_{4}$ (left) and the collection of spanning trees for $C_{4}$ (right).

Next, we compute the coefficients $b$ and $a$ involved in the formula of the group inverse. The corresponding spanning trees or rooted forests of any order are represented in Figures 10-13.

$$
\begin{aligned}
a_{1} & =4 \prod_{i=1}^{4} c_{i}\left(\frac{1}{w_{1}}+\frac{1}{w_{2}}+\frac{1}{w_{3}}+\frac{1}{w_{4}}\right) \\
a_{2} & =\prod_{i=1}^{4} c_{i}\left(\frac{\omega_{2}^{2}\left(\omega_{1}^{2}+\omega_{3}^{2}+\omega_{4}^{2}\right)}{w_{1} w_{2}}+\frac{\omega_{1}^{2}\left(\omega_{2}^{2}+\omega_{3}^{2}+\omega_{4}^{2}\right)}{w_{1} w_{4}}+\frac{\omega_{3}^{2}\left(\omega_{1}^{2}+\omega_{2}^{2}+\omega_{4}^{2}\right)}{w_{2} w_{3}}+\frac{\omega_{4}^{2}\left(\omega_{1}^{2}+\omega_{2}^{2}+\omega_{3}^{2}\right)}{w_{3} w_{4}}\right. \\
& \left.+\frac{\left(\omega_{2}^{2}+\omega_{3}^{2}\right)\left(\omega_{1}^{2}+\omega_{4}^{2}\right)}{w_{1} w_{3}}+\frac{\left(\omega_{3}^{2}+\omega_{4}^{2}\right)\left(\omega_{1}^{2}+\omega_{2}^{2}\right)}{w_{2} w_{4}}\right), \\
a_{3} & =\prod_{i=1}^{4} c_{i}\left(\frac{\omega_{2}^{2} \omega_{3}^{2}\left(\omega_{1}^{2}+\omega_{4}^{2}\right)}{w_{1} w_{2} w_{3}}+\frac{\omega_{1}^{2} \omega_{2}^{2}\left(\omega_{3}^{2}+\omega_{4}^{2}\right)}{w_{1} w_{2} w_{4}}+\frac{\omega_{1}^{2} \omega_{4}^{2}\left(\omega_{2}^{2}+\omega_{3}^{2}\right)}{w_{1} w_{3} w_{4}}+\frac{\omega_{3}^{2} \omega_{4}^{2}\left(\omega_{1}^{2}+\omega_{2}^{2}\right)}{w_{2} w_{3} w_{4}}\right), \\
a_{4} & =1 .
\end{aligned}
$$




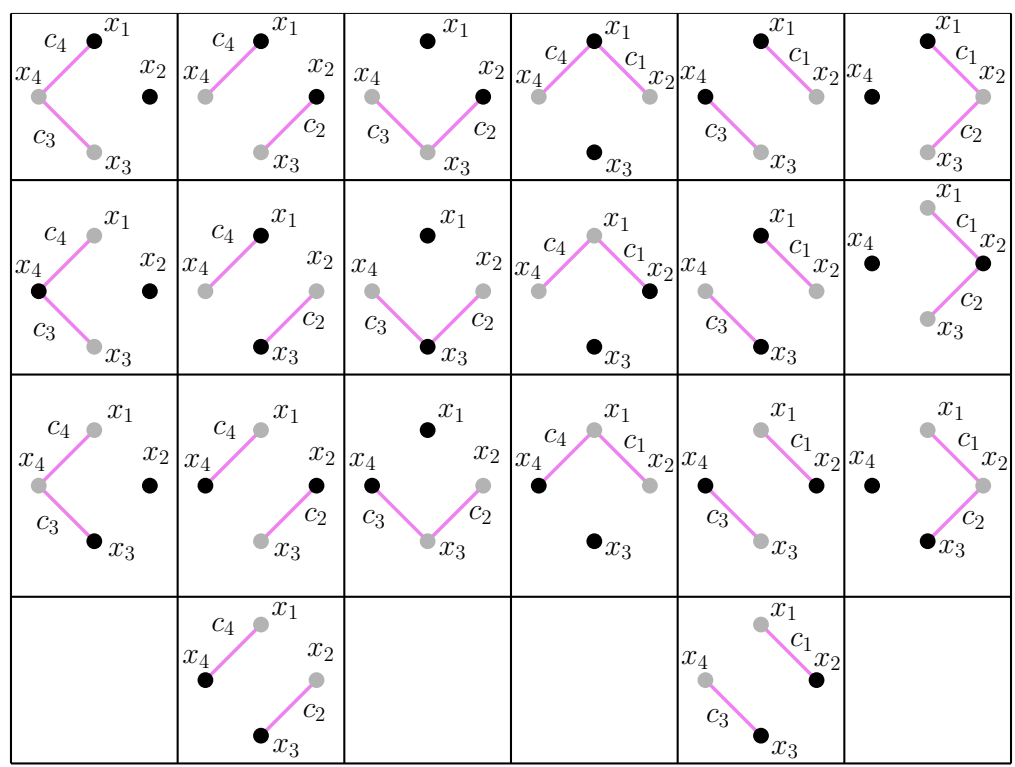

Figure 11: The collection of 2-rooted forests for $C_{4}$.

To compute the coefficient $b_{k}\left(x_{i}, x_{j}\right)$ it is enough to count the $k$-rooted forests displayed at Figures 11-13 with $x_{i}$ as a root and $x_{j}$ at the same component as $x_{i}$.

$$
\begin{aligned}
& b_{1}\left(x_{i}, x_{j}\right)=\frac{a_{1}}{4}, \\
& b_{2}\left(x_{1}, x_{1}\right)=\prod_{i=1}^{4} c_{i}\left(\frac{\omega_{2}^{2}}{w_{1} w_{2}}+\frac{\omega_{3}^{2}}{w_{2} w_{3}}+\frac{\omega_{4}^{2}}{w_{3} w_{4}}+\frac{\left(\omega_{2}^{2}+\omega_{3}^{2}\right)}{w_{1} w_{3}}+\frac{\left(\omega_{3}^{2}+\omega_{4}^{2}\right)}{w_{2} w_{4}}+\frac{\left(\omega_{2}^{2}+\omega_{3}^{2}+\omega_{4}^{2}\right)}{w_{1} w_{4}}\right), \\
& b_{2}\left(x_{2}, x_{2}\right)=\prod_{i=1}^{4} c_{i}\left(\frac{\omega_{1}^{2}}{w_{1} w_{4}}+\frac{\omega_{3}^{2}}{w_{2} w_{3}}+\frac{\omega_{4}^{2}}{w_{3} w_{4}}+\frac{\left(\omega_{1}^{2}+\omega_{4}^{2}\right)}{w_{1} w_{3}}+\frac{\left(\omega_{3}^{2}+\omega_{4}^{2}\right)}{w_{2} w_{4}}+\frac{\left(\omega_{1}^{2}+\omega_{3}^{2}+\omega_{4}^{2}\right)}{w_{1} w_{2}}\right), \\
& b_{2}\left(x_{3}, x_{3}\right)=\prod_{i=1}^{4} c_{i}\left(\frac{\omega_{1}^{2}}{w_{1} w_{4}}+\frac{\omega_{2}^{2}}{w_{1} w_{2}}+\frac{\omega_{4}^{2}}{w_{3} w_{4}}+\frac{\left(\omega_{1}^{2}+\omega_{4}^{2}\right)}{w_{1} w_{3}}+\frac{\left(\omega_{1}^{2}+\omega_{2}^{2}\right)}{w_{2} w_{4}}+\frac{\left(\omega_{1}^{2}+\omega_{2}^{2}+\omega_{4}^{2}\right)}{w_{2} w_{3}}\right), \\
& b_{2}\left(x_{4}, x_{4}\right)=\prod_{i=1}^{4} c_{i}\left(\frac{\omega_{1}^{2}}{w_{1} w_{4}}+\frac{\omega_{2}^{2}}{w_{1} w_{2}}+\frac{\omega_{3}^{2}}{w_{2} w_{3}}+\frac{\left(\omega_{1}^{2}+\omega_{2}^{2}\right)}{w_{2} w_{4}}+\frac{\left(\omega_{2}^{2}+\omega_{3}^{2}\right)}{w_{1} w_{3}}+\frac{\left(\omega_{1}^{2}+\omega_{2}^{2}+\omega_{3}^{2}\right)}{w_{3} w_{4}}\right), \\
& b_{2}\left(x_{1}, x_{2}\right)=\prod_{i=1}^{4} c_{i}\left(\frac{\omega_{3}^{2}}{w_{2} w_{3}}+\frac{\omega_{4}^{2}}{w_{3} w_{4}}+\frac{\left(\omega_{3}^{2}+\omega_{4}^{2}\right)}{w_{2} w_{4}}\right), \\
& b_{2}\left(x_{1}, x_{3}\right)=\prod_{i=1}^{4} c_{i}\left(\frac{\omega_{2}^{2}}{w_{1} w_{2}}+\frac{\omega_{4}^{2}}{w_{3} w_{4}}\right), \\
& b_{2}\left(x_{1}, x_{4}\right)=\prod_{i=1}^{4} c_{i}\left(\frac{\omega_{2}^{2}}{w_{1} w_{2}}+\frac{\omega_{3}^{2}}{w_{2} w_{3}}+\frac{\left(\omega_{2}^{2}+\omega_{3}^{2}\right)}{w_{1} w_{3}}\right), \\
& b_{2}\left(x_{3}, x_{4}\right)=\prod_{i=1}^{4} c_{i}\left(\frac{\omega_{1}^{2}}{w_{1} w_{4}}+\frac{\omega_{2}^{2}}{w_{1} w_{2}}+\frac{\left(\omega_{1}^{2}+\omega_{2}^{2}\right)}{w_{2} w_{4}}\right), \\
& b_{2}\left(x_{2}, x_{3}\right)=\prod_{i=1}^{4} c_{i}\left(\frac{\omega_{1}^{2}}{w_{1} w_{4}}+\frac{\omega_{4}^{2}}{w_{3} w_{4}}+\frac{\left(\omega_{1}^{2}+\omega_{4}^{2}\right)}{w_{1} w_{3}}\right), \\
& \left.b_{1} x_{4}\right)=\prod_{i}\left(\frac{\omega_{1}^{2}}{w_{1} w_{4}}+\frac{\omega_{3}^{2}}{w_{2} w_{3}}\right),
\end{aligned}
$$




$$
\begin{aligned}
& b_{3}\left(x_{1}, x_{1}\right)=\prod_{i=1}^{4} c_{i}\left(\frac{\omega_{2}^{2} \omega_{3}^{2}}{w_{1} w_{2} w_{3}}+\frac{\omega_{2}^{2}\left(\omega_{3}^{2}+\omega_{4}^{2}\right)}{w_{1} w_{2} w_{4}}+\frac{\omega_{2}^{2} \omega_{3}^{2}}{w_{1} w_{2} w_{3}}+\frac{\omega_{4}^{2}\left(\omega_{2}^{2}+\omega_{3}^{2}\right)}{w_{2} w_{3} w_{4}}\right), \\
& b_{3}\left(x_{2}, x_{2}\right)=\prod_{i=1}^{4} c_{i}\left(\frac{\omega_{1}^{2} \omega_{4}^{2}}{w_{1} w_{2} w_{3}}+\frac{\omega_{1}^{2}\left(\omega_{3}^{2}+\omega_{4}^{2}\right)}{w_{1} w_{2} w_{4}}+\frac{\omega_{3}^{2} \omega_{4}^{2}}{w_{2} w_{3} w_{4}}+\frac{\omega_{3}^{2}\left(\omega_{1}^{2}+\omega_{4}^{2}\right)}{w_{1} w_{2} w_{3}}\right), \\
& b_{3}\left(x_{3}, x_{3}\right)=\prod_{i=1}^{4} c_{i}\left(\frac{\omega_{1}^{2} \omega_{4}^{2}}{w_{1} w_{3} w_{4}}+\frac{\omega_{4}^{2}\left(\omega_{1}^{2}+\omega_{2}^{2}\right)}{w_{2} w_{3} w_{4}}+\frac{\omega_{1}^{2} \omega_{2}^{2}}{w_{1} w_{2} w_{4}}+\frac{\omega_{2}^{2}\left(\omega_{1}^{2}+\omega_{4}^{2}\right)}{w_{1} w_{2} w_{3}}\right), \\
& b_{3}\left(x_{4}, x_{4}\right)=\prod_{i=1}^{4} c_{i}\left(\frac{\omega_{1}^{2} \omega_{2}^{2}}{w_{1} w_{2} w_{4}}+\frac{\omega_{3}^{2}\left(\omega_{1}^{2}+\omega_{2}^{2}\right)}{w_{2} w_{3} w_{4}}+\frac{\omega_{2}^{2} \omega_{3}^{2}}{w_{1} w_{2} w_{3}}+\frac{\omega_{1}^{2}\left(\omega_{2}^{2}+\omega_{3}^{2}\right)}{w_{1} w_{3} w_{4}}\right), \\
& b_{3}\left(x_{1}, x_{3}\right)=b_{3}\left(x_{2}, x_{4}\right)=0, \\
& b_{3}\left(x_{1}, x_{4}\right)=\prod_{i=1}^{4} c_{i} \frac{\omega_{2}^{2} \omega_{3}^{2}}{w_{1} w_{2} w_{3}}, \quad b_{3}\left(x_{2}, x_{3}\right)=\prod_{i=1}^{4} c_{i} \frac{\omega_{1}^{2} \omega_{4}^{2}}{w_{1} w_{3} w_{4}}, \\
& b_{4}\left(x_{i}, x_{i}\right)=\frac{1}{\omega_{i}^{2}}, \quad b_{3}\left(x_{3}, x_{4}\right)=\prod_{i=1}^{4} c_{i} \frac{\omega_{1}^{2} \omega_{2}^{2}}{w_{1} w_{2} w_{4}},
\end{aligned}
$$




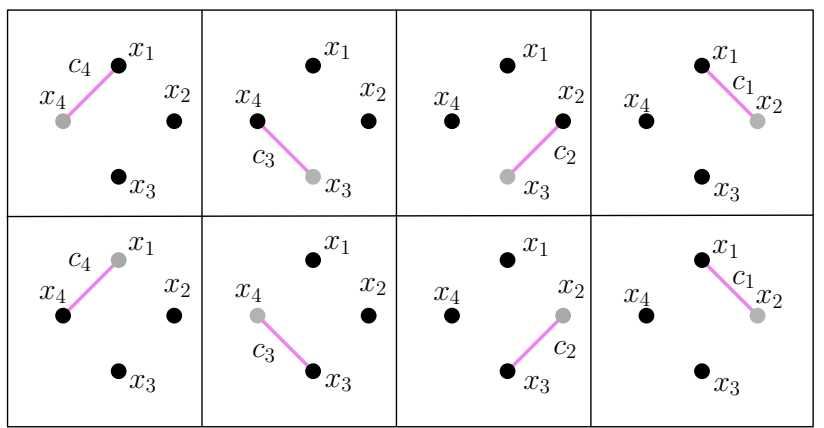

Figure 12: The collection of 3-rooted forests for $C_{4}$.

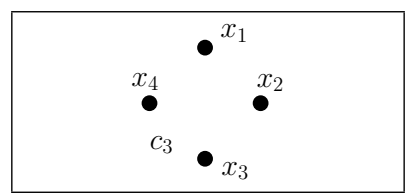

Figure 13: The 4-rooted forest for $C_{4}$.

Hence, we could compute the term involved in the expression for the Green function and the inverse of $\mathrm{L}_{\mathrm{q}}$ for $C_{4}$. However, due to the length of these expressions we only give explicitly the Green kernel for the case of $\lambda=0$.

$$
\begin{aligned}
& 4 b_{2}\left(x_{1}, x_{1}\right)-a_{2}=\prod_{i=1}^{4} c_{i}\left(\frac{\omega_{2}^{4}}{w_{1} w_{2}}+\frac{\omega_{3}^{4}}{w_{2} w_{3}}+\frac{\omega_{4}^{4}}{w_{3} w_{4}}+\frac{\left(\omega_{2}^{2}+\omega_{3}^{2}\right)^{2}}{w_{1} w_{3}}+\frac{\left(\omega_{3}^{2}+\omega_{4}^{2}\right)^{2}}{w_{2} w_{4}}+\frac{\left(\omega_{2}^{2}+\omega_{3}^{2}+\omega_{4}^{2}\right)^{2}}{w_{1} w_{4}}\right), \\
& 4 b_{2}\left(x_{2}, x_{2}\right)-a_{2}=\prod_{i=1}^{4} c_{i}\left(\frac{\omega_{1}^{4}}{w_{1} w_{4}}+\frac{\omega_{3}^{4}}{w_{2} w_{3}}+\frac{\omega_{4}^{4}}{w_{3} w_{4}}+\frac{\left(\omega_{1}^{2}+\omega_{4}^{2}\right)^{2}}{w_{1} w_{3}}+\frac{\left(\omega_{3}^{2}+\omega_{4}^{2}\right)^{2}}{w_{2} w_{4}}+\frac{\left(\omega_{1}^{2}+\omega_{3}^{2}+\omega_{4}^{2}\right)^{2}}{w_{1} w_{2}}\right), \\
& 4 b_{2}\left(x_{3}, x_{3}\right)-a_{2}=\prod_{i=1}^{4} c_{i}\left(\frac{\omega_{1}^{4}}{w_{1} w_{4}}+\frac{\omega_{2}^{4}}{w_{1} w_{2}}+\frac{\omega_{4}^{4}}{w_{3} w_{4}}+\frac{\left(\omega_{1}^{2}+\omega_{4}^{2}\right)^{2}}{w_{1} w_{3}}+\frac{\left(\omega_{1}^{2}+\omega_{2}^{2}\right)^{2}}{w_{2} w_{4}}+\frac{\left(\omega_{1}^{2}+\omega_{2}^{2}+\omega_{4}^{2}\right)^{2}}{w_{2} w_{3}}\right), \\
& 4 b_{2}\left(x_{4}, x_{4}\right)-a_{2}=\prod_{i=1}^{4} c_{i}\left(\frac{\omega_{1}^{4}}{w_{1} w_{4}}+\frac{\omega_{2}^{4}}{w_{1} w_{2}}+\frac{\omega_{3}^{4}}{w_{2} w_{3}}+\frac{\left(\omega_{2}^{2}+\omega_{3}^{2}\right)^{2}}{w_{1} w_{3}}+\frac{\left(\omega_{1}^{2}+\omega_{2}^{2}\right)^{2}}{w_{2} w_{4}}+\frac{\left(\omega_{1}^{2}+\omega_{2}^{2}+\omega_{3}^{2}\right)^{2}}{w_{3} w_{4}}\right),
\end{aligned}
$$




$$
\begin{aligned}
& 4 b_{2}\left(x_{1}, x_{2}\right)-a_{2}=\prod_{i=1}^{4} c_{i}\left(\frac{\omega_{3}^{4}}{w_{2} w_{3}}+\frac{\omega_{4}^{4}}{w_{3} w_{4}}+\frac{\left(\omega_{3}^{2}+\omega_{4}^{2}\right)^{2}}{w_{2} w_{4}}-\frac{\left(\omega_{2}^{2}+\omega_{3}^{2}\right)\left(\omega_{1}^{2}+\omega_{4}^{2}\right)}{w_{1} w_{3}}\right. \\
& \left.-\frac{\omega_{2}^{2}\left(\omega_{1}^{2}+\omega_{3}^{2}+\omega_{4}^{2}\right)}{w_{1} w_{2}}-\frac{\omega_{1}^{2}\left(\omega_{2}^{2}+\omega_{3}^{2}+\omega_{4}^{2}\right)}{w_{1} w_{4}}\right), \\
& 4 b_{2}\left(x_{1}, x_{3}\right)-a_{2}=\prod_{i=1}^{4} c_{i}\left(\frac{\omega_{2}^{4}}{w_{1} w_{2}}+\frac{\omega_{4}^{4}}{w_{3} w_{4}}-\frac{\omega_{1}^{2}\left(\omega_{2}^{2}+\omega_{3}^{2}+\omega_{4}^{2}\right)}{w_{1} w_{4}}-\frac{\omega_{3}^{2}\left(\omega_{1}^{2}+\omega_{2}^{2}+\omega_{4}^{2}\right)}{w_{2} w_{3}}\right. \\
& \left.-\frac{\left(\omega_{2}^{2}+\omega_{3}^{2}\right)\left(\omega_{1}^{2}+\omega_{4}^{2}\right)}{w_{1} w_{3}}-\frac{\left(\omega_{3}^{2}+\omega_{4}^{2}\right)\left(\omega_{1}^{2}+\omega_{2}^{2}\right)}{w_{2} w_{4}}\right) \\
& 4 b_{2}\left(x_{1}, x_{4}\right)-a_{2}=\prod_{i=1}^{4} c_{i}\left(\frac{\omega_{2}^{4}}{w_{1} w_{2}}+\frac{\omega_{3}^{4}}{w_{2} w_{3}}+\frac{\left(\omega_{2}^{2}+\omega_{3}^{2}\right)^{2}}{w_{1} w_{3}}-\frac{\omega_{1}^{2}\left(\omega_{2}^{2}+\omega_{3}^{2}+\omega_{4}^{2}\right)}{w_{1} w_{4}}\right. \\
& \left.-\frac{\omega_{4}^{2}\left(\omega_{1}^{2}+\omega_{2}^{2}+\omega_{3}^{2}\right)}{w_{3} w_{4}}-\frac{\left(\omega_{3}^{2}+\omega_{4}^{2}\right)\left(\omega_{1}^{2}+\omega_{2}^{2}\right)}{w_{2} w_{4}}\right), \\
& 4 b_{2}\left(x_{2}, x_{3}\right)-a_{2}=\prod_{i=1}^{4} c_{i}\left(\frac{\omega_{1}^{4}}{w_{1} w_{4}}+\frac{\omega_{4}^{4}}{w_{3} w_{4}}+\frac{\left(\omega_{1}^{2}+\omega_{4}^{2}\right)^{2}}{w_{1} w_{3}}-\frac{\omega_{2}^{2}\left(\omega_{1}^{2}+\omega_{3}^{2}+\omega_{4}^{2}\right)}{w_{1} w_{2}}\right. \\
& \left.-\frac{\omega_{3}^{2}\left(\omega_{1}^{2}+\omega_{2}^{2}+\omega_{4}^{2}\right)}{w_{2} w_{3}}-\frac{\left(\omega_{3}^{2}+\omega_{4}^{2}\right)\left(\omega_{1}^{2}+\omega_{2}^{2}\right)}{w_{2} w_{4}}\right), \\
& 4 b_{2}\left(x_{2}, x_{4}\right)-a_{2}=\prod_{i=1}^{4} c_{i}\left(\frac{\omega_{1}^{4}}{w_{1} w_{4}}+\frac{\omega_{3}^{4}}{w_{2} w_{3}}-\frac{\omega_{2}^{2}\left(\omega_{1}^{2}+\omega_{3}^{2}+\omega_{4}^{2}\right)}{w_{1} w_{2}}-\frac{\omega_{4}^{2}\left(\omega_{1}^{2}+\omega_{2}^{2}+\omega_{3}^{2}\right)}{w_{3} w_{4}}\right. \\
& \left.-\frac{\left(\omega_{2}^{2}+\omega_{3}^{2}\right)\left(\omega_{1}^{2}+\omega_{4}^{2}\right)}{w_{1} w_{3}}-\frac{\left(\omega_{3}^{2}+\omega_{4}^{2}\right)\left(\omega_{1}^{2}+\omega_{2}^{2}\right)}{w_{2} w_{4}}\right) \\
& 4 b_{2}\left(x_{3}, x_{4}\right)-a_{2}=\prod_{i=1}^{4} c_{i}\left(\frac{\omega_{1}^{4}}{w_{1} w_{4}}+\frac{\omega_{2}^{4}}{w_{1} w_{2}}-\frac{\left(\omega_{1}^{2}+\omega_{2}^{2}\right)^{2}}{w_{2} w_{4}}-\frac{\omega_{3}^{2}\left(\omega_{1}^{2}+\omega_{2}^{2}+\omega_{4}^{2}\right)}{w_{2} w_{3}}\right. \\
& \left.-\frac{\omega_{4}^{2}\left(\omega_{1}^{2}+\omega_{2}^{2}+\omega_{3}^{2}\right)}{w_{3} w_{4}}-\frac{\left(\omega_{2}^{2}+\omega_{3}^{2}\right)\left(\omega_{1}^{2}+\omega_{4}^{2}\right)}{w_{1} w_{3}}\right) .
\end{aligned}
$$

Therefore, using again the expression obtained in Proposition 4.1, we get the Green function for $C_{4}$ and $\lambda=0$. 
$\frac{G\left(x_{1}, x_{1}\right)}{\omega_{1}^{2}}=\frac{\frac{\omega_{2}^{4}}{w_{1} w_{2}}+\frac{\omega_{3}^{4}}{w_{2} w_{3}}+\frac{\omega_{4}^{4}}{w_{3} w_{4}}+\frac{\left(\omega_{2}^{2}+\omega_{3}^{2}\right)^{2}}{w_{1} w_{3}}+\frac{\left(\omega_{3}^{2}+\omega_{4}^{2}\right)^{2}}{w_{2} w_{4}}+\frac{\left(\omega_{2}^{2}+\omega_{3}^{2}+\omega_{4}^{2}\right)^{2}}{w_{1} w_{4}}}{16\left(\frac{1}{w_{1}}+\frac{1}{w_{2}}+\frac{1}{w_{3}}+\frac{1}{w_{4}}\right)}$,

$\frac{G\left(x_{2}, x_{2}\right)}{\omega_{2}^{2}}=\frac{\frac{\omega_{1}^{4}}{w_{1} w_{4}}+\frac{\omega_{3}^{4}}{w_{2} w_{3}}+\frac{\omega_{4}^{4}}{w_{3} w_{4}}+\frac{\left(\omega_{1}^{2}+\omega_{4}^{2}\right)^{2}}{w_{1} w_{3}}+\frac{\left(\omega_{3}^{2}+\omega_{4}^{2}\right)^{2}}{w_{2} w_{4}}+\frac{\left(\omega_{1}^{2}+\omega_{3}^{2}+\omega_{4}^{2}\right)^{2}}{w_{1} w_{2}}}{16\left(\frac{1}{w_{1}}+\frac{1}{w_{2}}+\frac{1}{w_{3}}+\frac{1}{w_{4}}\right)}$,

$\frac{G\left(x_{3}, x_{3}\right)}{\omega_{3}^{2}}=\frac{\frac{\omega_{1}^{4}}{w_{1} w_{4}}+\frac{\omega_{2}^{4}}{w_{1} w_{2}}+\frac{\omega_{4}^{4}}{w_{3} w_{4}}+\frac{\left(\omega_{1}^{2}+\omega_{4}^{2}\right)^{2}}{w_{1} w_{3}}+\frac{\left(\omega_{1}^{2}+\omega_{2}^{2}\right)^{2}}{w_{2} w_{4}}+\frac{\left(\omega_{1}^{2}+\omega_{2}^{2}+\omega_{4}^{2}\right)^{2}}{w_{2} w_{3}}}{16\left(\frac{1}{w_{1}}+\frac{1}{w_{2}}+\frac{1}{w_{3}}+\frac{1}{w_{4}}\right)}$,

$\frac{G\left(x_{4}, x_{4}\right)}{\omega_{4}^{2}}=\frac{\frac{\omega_{1}^{4}}{w_{1} w_{4}}+\frac{\omega_{2}^{4}}{w_{1} w_{2}}+\frac{\omega_{3}^{4}}{w_{2} w_{3}}+\frac{\left(\omega_{2}^{2}+\omega_{3}^{2}\right)^{2}}{w_{1} w_{3}}+\frac{\left(\omega_{1}^{2}+\omega_{2}^{2}\right)^{2}}{w_{2} w_{4}}+\frac{\left(\omega_{1}^{2}+\omega_{2}^{2}+\omega_{3}^{2}\right)^{2}}{w_{3} w_{4}}}{16\left(\frac{1}{w_{1}}+\frac{1}{w_{2}}+\frac{1}{w_{3}}+\frac{1}{w_{4}}\right)}$,

$\frac{G\left(x_{1}, x_{2}\right)}{\omega_{1} \omega_{2}}=\frac{\frac{\omega_{3}^{4}}{w_{2} w_{3}}+\frac{\omega_{4}^{4}}{w_{3} w_{4}}+\frac{\left(\omega_{3}^{2}+\omega_{4}^{2}\right)^{2}}{w_{2} w_{4}}-\frac{\left(\omega_{2}^{2}+\omega_{3}^{2}\right)\left(\omega_{1}^{2}+\omega_{4}^{2}\right)}{w_{1} w_{3}}-\frac{\omega_{2}^{2}\left(\omega_{1}^{2}+\omega_{3}^{2}+\omega_{4}^{2}\right)}{w_{1} w_{2}}-\frac{\omega_{1}^{2}\left(\omega_{2}^{2}+\omega_{3}^{2}+\omega_{4}^{2}\right)}{w_{1} w_{4}}}{16\left(\frac{1}{w_{1}}+\frac{1}{w_{2}}+\frac{1}{w_{3}}+\frac{1}{w_{4}}\right)}$,

$\frac{G\left(x_{1}, x_{3}\right)}{\omega_{1} \omega_{3}}=\frac{\frac{\omega_{2}^{4}}{w_{1} w_{2}}+\frac{\omega_{4}^{4}}{w_{3} w_{4}}-\frac{\omega_{1}^{2}\left(\omega_{2}^{2}+\omega_{3}^{2}+\omega_{4}^{2}\right)}{w_{1} w_{4}}-\frac{\omega_{3}^{2}\left(\omega_{1}^{2}+\omega_{2}^{2}+\omega_{4}^{2}\right)}{w_{2} w_{3}}-\frac{\left(\omega_{2}^{2}+\omega_{3}^{2}\right)\left(\omega_{1}^{2}+\omega_{4}^{2}\right)}{w_{1} w_{3}}-\frac{\left(\omega_{3}^{2}+\omega_{4}^{2}\right)\left(\omega_{1}^{2}+\omega_{2}^{2}\right)}{w_{2} w_{4}},}{16\left(\frac{1}{w_{1}}+\frac{1}{w_{2}}+\frac{1}{w_{3}}+\frac{1}{w_{4}}\right)}$,

$\frac{G\left(x_{1}, x_{4}\right)}{\omega_{1} \omega_{4}}=\frac{\frac{\omega_{2}^{4}}{w_{1} w_{2}}+\frac{\omega_{3}^{4}}{w_{2} w_{3}}+\frac{\left(\omega_{2}^{2}+\omega_{3}^{2}\right)^{2}}{w_{1} w_{3}}-\frac{\omega_{1}^{2}\left(\omega_{2}^{2}+\omega_{3}^{2}+\omega_{4}^{2}\right)}{w_{1} w_{4}}-\frac{\omega_{4}^{2}\left(\omega_{1}^{2}+\omega_{2}^{2}+\omega_{3}^{2}\right)}{w_{3} w_{4}}-\frac{\left(\omega_{3}^{2}+\omega_{4}^{2}\right)\left(\omega_{1}^{2}+\omega_{2}^{2}\right)}{w_{2} w_{4}},}{16\left(\frac{1}{w_{1}}+\frac{1}{w_{2}}+\frac{1}{w_{3}}+\frac{1}{w_{4}}\right)}$,

$\frac{G\left(x_{2}, x_{3}\right)}{\omega_{2} \omega_{3}}=\frac{\frac{\omega_{1}^{4}}{w_{1} w_{4}}+\frac{\omega_{4}^{4}}{w_{3} w_{4}}+\frac{\left(\omega_{1}^{2}+\omega_{4}^{2}\right)^{2}}{w_{1} w_{3}}-\frac{\omega_{2}^{2}\left(\omega_{1}^{2}+\omega_{3}^{2}+\omega_{4}^{2}\right)}{w_{1} w_{2}}-\frac{\omega_{3}^{2}\left(\omega_{1}^{2}+\omega_{2}^{2}+\omega_{4}^{2}\right)}{w_{2} w_{3}}-\frac{\left(\omega_{3}^{2}+\omega_{4}^{2}\right)\left(\omega_{1}^{2}+\omega_{2}^{2}\right)}{w_{2} w_{4}}}{16\left(\frac{1}{w_{1}}+\frac{1}{w_{2}}+\frac{1}{w_{3}}+\frac{1}{w_{4}}\right)}$,

$\frac{G\left(x_{2}, x_{4}\right)}{\omega_{2} \omega_{4}}=\frac{\frac{\omega_{1}^{4}}{w_{1} w_{4}}+\frac{\omega_{3}^{4}}{w_{2} w_{3}}-\frac{\omega_{2}^{2}\left(\omega_{1}^{2}+\omega_{3}^{2}+\omega_{4}^{2}\right)}{w_{1} w_{2}}-\frac{\omega_{4}^{2}\left(\omega_{1}^{2}+\omega_{2}^{2}+\omega_{3}^{2}\right)}{w_{3} w_{4}}-\frac{\left(\omega_{2}^{2}+\omega_{3}^{2}\right)\left(\omega_{1}^{2}+\omega_{4}^{2}\right)}{w_{1} w_{3}}-\frac{\left(\omega_{3}^{2}+\omega_{4}^{2}\right)\left(\omega_{1}^{2}+\omega_{2}^{2}\right)}{w_{2} w_{4}},}{16\left(\frac{1}{w_{1}}+\frac{1}{w_{2}}+\frac{1}{w_{3}}+\frac{1}{w_{4}}\right)}$,

$\frac{G\left(x_{3}, x_{4}\right)}{\omega_{3} \omega_{4}}=\frac{\frac{\omega_{1}^{4}}{w_{1} w_{4}}+\frac{\omega_{2}^{4}}{w_{1} w_{2}}+\frac{\left(\omega_{1}^{2}+\omega_{2}^{2}\right)^{2}}{w_{2} w_{4}}-\frac{\omega_{3}^{2}\left(\omega_{1}^{2}+\omega_{2}^{2}+\omega_{4}^{2}\right)}{w_{2} w_{3}}-\frac{\omega_{4}^{2}\left(\omega_{1}^{2}+\omega_{2}^{2}+\omega_{3}^{2}\right)}{w_{3} w_{4}}-\frac{\left(\omega_{2}^{2}+\omega_{3}^{2}\right)\left(\omega_{1}^{2}+\omega_{4}^{2}\right)}{w_{1} w_{3}}}{16\left(\frac{1}{w_{1}}+\frac{1}{w_{2}}+\frac{1}{w_{3}}+\frac{1}{w_{4}}\right)}$. 
Acknowledgments: This work has been partly supported by the Spanish Research Council (Comisión Interministerial de Ciencia y Tecnología,) under project MTM2017-85996-R.

Data Availability Statement: Data sharing is not applicable to this article as no datasets were generated or analyzed during the current study.

\section{References}

[1] A. Abdesselam, The Grassmann-Berezin calculus and theorems of the matrix-tree type, Ad. Appl. Math. 33 (2004) 51-70.

[2] E. Bendito, A. Carmona and A. M. Encinas, Potential theory for Schrödinger operators on finite networks, Rev. Mat. Iberoamericana 21 (2005) 771-818.

[3] E. Bendito, A. Carmona and A. M. Encinas, Eigenvalues, eigenfuntions and Green's functions on a path via Chebyshev polynomials, Appl. Anal. Discrete Math. 3 (2009) 282-302.

[4] E. Bendito, A. Carmona, A.M. Encinas and J.M. Gesto, Characterization of symmetric $M$-matrices as resistive inverses, Linear Algebra Appl. 430 (2009) 1336-1349.

[5] E. Bendito, A. Carmona, A.M. Encinas and M. Mitjana, The $M$-matrix inverse problem for singular and symmetric Jacobi matrices, Linear Algebra Appl. 436 (2012) 1090-1098.

[6] T. Biyikoğlu, J. Leydold and P.F. Stadler, Laplacian Eigenvectors of Graphs, LNM 1915, Springer, Berlin, 2007.

[7] B. Bollobás, Modern graph theory, Graduate Texts in Mathematics, 184, Springer-Verlag, New York, 1998

[8] Y. Burman and B. Shapiro, Around matrix-tree theorem, Math. Res. Lett. 13 (2006) 761-774.

[9] A. Carmona, A.M. Encinas and M. Mitjana, Discrete elliptic operators and their Green operators, Linear Algebra Appl. 442 (2014) 115-134.

[10] A. Carmona, A.M. Encinas and M. Mitjana, Resistance distances on networks, Appl. Anal. Discrete Math. 11 (2017) $136-147$.

[11] S. Chaiken, A combinatorial proof of the all minors matrix tree theorem, SIAM J. Alg. Disc. Math. 3 (1982) 319-329.

[12] P. Chebotarev and E. Shamis, On proximity measures for graphs vertices, Automat. Remote Control 59 (1998) $1443-1459$.

[13] P. Chebotarev and R. Agaev, Forest matrices around the Laplacian matrix, Linear Algebra Appl. 356 (2002) $253-274$.

[14] P. Chebotarev, Spanning forests and the golden ratio, Discrete Appl. Math. 156, (2008) 813-821.

[15] P. Chebotarev and E. Shamis, The Forest Metrics for Graph Vertices, Electron. Notes Discrete Math. 11 (2002) 98-107.

[16] F. Chung, and W. Zhao, PageRank and random walks on graphs, Fete of combinatorics and computer science, Bolyai Soc. Math. Stud., 20 János Bolyai Math. Soc., Budapest, 2010, 43-62.

[17] C. Dellacherie, S. Martínez and J. San Martín, Ultrametric matrices and induced Markov Chains, Adv. Appl. Math. 17 (1996) 169-183.

[18] C. Dellacherie, S. Martínez and J. San Martín, Description of the sub-Markov kernel associated to generalized ultrametric matrices. An algorithm approach, Linear Algebra Appl. 318 (2000) 1-21.

[19] A.M. Encinas and M.J. Jiménez, Second order linear difference equations, J. Difference Equ. Appl. 24 (2018) 305-343.

[20] M. Fiedler, Some characterizations of symmetric $M$-matrices, Linear Algebra Appl. 275-276 (1998) 179-187.

[21] D. Kalita, Determinant of the Laplacian Matrix of a Weighted Directed Graph, in Combinatorial Matrix Theory and Generalized Inverses of Matrices, R.R. Bapat, S.J. Kirkland, K.M. Prasad and S. Puntanen, Eds. Springer (2013).

[22] G. Kirchhoff, Über die Auflösung der Gleichungen, auf welche man bei der Untersuchung der linearen Verteilung galvanischer Ströme gefuhrt wird, Ann. Phys. Chem. 72 (1847) 497-508.

[23] S. Kirkland and M. Neumann, Group inverses of M-matrices and their applications, Chapman \& Hall/CRC Applied Mathematics and Nonlinear Science Series, CRC Press, Boca Raton, FL, 2013.

[24] S. Kirkland, M. Neumann and B. Shader, Distances in weighted trees and group inverse of Laplacian matrices, SIAM J. Matrix Anal. Appl., 18 (1997) 827-841.

[25] S. Klee and M. Stamps, Linear algebraic techniques for weighted spanning tree enumeration, Linear Algebra Appl.582 (2019) 391-402.

[26] 0. Knill, Counting rooted forests in a network, available in: arXiv:1307.3810v2 [math.SP].

[27] O. Knill, Cauchy-Binet for pseudo-determinants, Linear Algebra Appl. 459 (2014) 522-547.

[28] J. Zhou and C. Bu, The enumeration of spanning tree of weighted graphs, J. Algebaric Combin., DOI: 10.1007/s10801-02000969-w. 